\title{
Broadly neutralizing anti-S2 antibodies protect against all three human betacoronaviruses that cause severe disease
}

Panpan Zhou ${ }^{1,2,3 \dagger}$, Ge Song1,2,3†, Wan-ting He $\mathrm{He}^{1,2,3}$, Nathan Beutler ${ }^{1}$, Longping V. Tse ${ }^{4}$, David R. Martinez ${ }^{4}$, Alexandra Schäfer ${ }^{4}$, Fabio Anzanello ${ }^{1,2,3}$, Peter Yong 1,2,3, Linghang Peng $^{1}$, Katharina Dueker ${ }^{1,2,3}$, Rami Musharrafieh ${ }^{1,2,3}$, Sean Callaghan ${ }^{1,2,3}$, Tazio

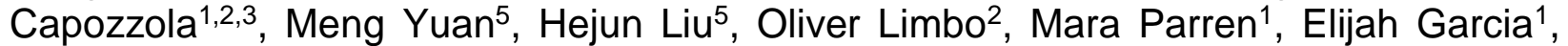
Stephen A. Rawlings ${ }^{6}$, Davey M. Smith ${ }^{6}$, David Nemazee ${ }^{1}$, Joseph G. Jardine ${ }^{2}$, Ian A.

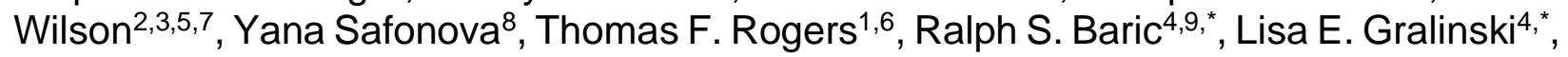

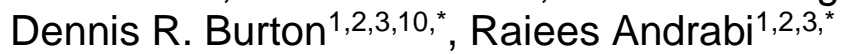

${ }^{1}$ Department of Immunology and Microbiology, The Scripps Research Institute, La Jolla, CA 92037, USA.

${ }^{2}$ IAVI Neutralizing Antibody Center, The Scripps Research Institute, La Jolla, CA 92037, USA

${ }^{3}$ Consortium for HIV/AIDS Vaccine Development (CHAVD), The Scripps Research Institute, La Jolla, CA 92037, USA.

${ }^{4}$ Department of Epidemiology, The University of North Carolina at Chapel Hill, Chapel Hill, NC 27599, USA.

${ }^{5}$ Department of Integrative Structural and Computational Biology, The Scripps Research Institute, La Jolla, CA 92037, USA.

${ }^{6}$ Division of Infectious Diseases, Department of Medicine, University of California, San Diego, La Jolla, CA 92037, USA.

${ }^{7}$ Skaggs Institute for Chemical Biology, The Scripps Research Institute, La Jolla, CA 92037, USA

${ }^{8}$ Department of Computer Science, Johns Hopkins University, Baltimore, MD 21218 , USA

${ }^{9}$ Departments of Microbiology and Immunology, The University of North Carolina at Chapel Hill, Chapel Hill, NC 27599, USA.

${ }^{10}$ Ragon Institute of Massachusetts General Hospital, Massachusetts Institute of Technology, and Harvard University, Cambridge, MA 02139, USA.

†These authors contributed equally to this work.

*Corresponding author. Email: rbaric@email.unc.edu (R.S.B.); Igralins@email.unc.edu

34 (L.E.G.); burton@scripps.edu (D.R.B.); andrabi@scripps.edu (R.A.). 
Abstract

Pan-betacoronavirus neutralizing antibodies may hold the key to developing broadly protective vaccines against coronaviruses that cause severe disease, for anticipating novel pandemic-causing viruses, and to respond more effectively to SARS-CoV-2 variants. The emergence of the Omicron variant of SARS-CoV-2 has illustrated the limitations of solely targeting the receptor binding domain (RBD) of the envelope Spike (S)-protein. Here, we isolated a large panel of broadly neutralizing antibodies (bnAbs) from SARS-CoV-2 recovered-vaccinated donors that target a conserved $\mathrm{S} 2$ region in the fusion machinery on betacoronavirus spikes. Select bnAbs show broad in vivo protection against all three pathogenic betacoronaviruses, SARS-CoV-1, SARS-CoV-2 and MERS-CoV, that have spilled over into humans in the past 20 years to cause severe disease. The bnAbs provide new opportunities for antibody-based interventions and key insights for developing pan-betacoronavirus vaccines. 


\section{Main}

The initial successes of SARS-CoV-2 vaccines were due in part to the relative ease of inducing protective neutralizing antibodies (nAbs) to immunodominant epitopes on the receptor binding domain (RBD) of the $S 1$ subunit of the spike protein (1-5). The most potent nAbs target epitopes overlapping the ACE2 receptor binding site (RBS) (RBS$\mathrm{A} /$ class 1 ; RBS-B,C,D/class 2 antibodies) and require little affinity maturation to neutralize at very low (single digit $\mathrm{ng} / \mathrm{ml}$ ) concentrations (3, 6-12). However, mutations, particularly in and around the RBS, readily generate viral variants that are resistant to neutralization by commonly induced classes of antibodies $(13,14)$ without significantly negatively impacting viral fitness, and have led to several Variants of Concern (VOCs) (14-25). For example, the $\mathrm{K} 417 \mathrm{~N} / \mathrm{T}$ and $\mathrm{E} 484 \mathrm{~K}$ mutations in the Beta and Gamma VOCs leads to neutralization escape from the vast majority of RBS-A/class 1 and RBS-B, C, D/class 2 nAbs (12-14). Other sarbecoviruses using ACE2 as receptor, such as SARS-CoV-1, and betacoronaviruses using receptors other than ACE2, such as MERS-CoV, show even more sequence divergence in the RBS region (26-28). The emergence of SARS-CoV-2 VOCs, together with a desire to have the capability to respond to novel coronaviruses with pandemic potential, has focused effort on vaccines and antibodies that target the most conserved regions of the spike protein (29-31). The "lower" more conserved faces of the RBD have been investigated and are targeted by many nAbs with greater breadth of neutralization against SARS-CoV-2 variants and diverse sarbecoviruses than for example RBS-A/class 1 or RBS-B,C,D/class 2 nAbs (32-41). However, the Omicron variant has demonstrated escape also from some nAbs targeting these more conserved regions of the $\operatorname{RBD}(19,25)$. An alternative relatively conserved target on the coronavirus spike is the S2 region, which does harbor neutralizing epitopes (42) and therefore is of interest for attempts to generate SARS-CoV-2 vaccines effective against VOCs and, more ambitiously, pan-betacoronavirus vaccines (29-31).

We recently isolated a $n A b, C C 40.8$, from a COVID-19 convalescent donor that neutralized sarbecoviruses from clades $1 \mathrm{a}$ and $1 \mathrm{~b}$ and SARS-CoV-2 VOCs $(43,44)$. We demonstrated antibody protection against SARS-CoV-2 challenge in human ACE2 mouse and hamster models (44). We further mapped the epitope of CC40.8 to the conserved spike S2 stem-helix region that forms part of the spike fusion machinery (44). Several more bnAbs targeting this region have been isolated from humans and from vaccinated animals (45-51). These bnAbs are a good starting point and highlight the opportunities that conserved bnAb S2 epitopes may offer for broad betacoronavirus vaccine targeting. However, a large panel of stem-helix bnAbs will be needed to define the common molecular features of antibodies targeting this site, which may facilitate the development of rational vaccine strategies that can induce such bnAbs by vaccination (52-56). Such a panel would also provide more options for antibody-based prophylaxis and therapeutic strategies (57). 


\section{Results}

\section{Donors for isolation of $\beta$-CoV spike stem-helix bnAbs}

To identify suitable donors for the isolation of a panel of $\beta$-CoV spike stem-helix bnAbs, we screened immune sera from human donors for cross-reactive binding to 25-mer spike stem-helix region peptides, which we previously identified as a target for bnAbs $(43,44)$. We tested sera from three different groups of donors: i) COVID-19 recovered donors (n $=15)$; ii) spike mRNA-vaccinated $(2 X)$ donors $(n=10)$ and iii) COVID-19-recovered then spike-vaccinated $(1 X)$ donors $(n=15)$ (Fig. 1a). Whereas weak or no binding was observed for COVID-19 recovered or vaccinee sera to human $\beta$-CoV spike stem-helix peptides, sera from $80 \%(12 / 15)$ of recovered-vaccinated donors exhibited strong crossreactive binding to the peptides (Fig. 1a). We noted a strong correlation between binding of recovered-vaccinated sera to SARS-CoV-2 stem-helix peptide with binding to other human $\beta$-CoV stem-helix peptides suggesting targeting of common cross-reactive epitopes (Fig. 1b). Accordingly, we sought to isolate $\beta$-CoV stem-helix directed bnAbs from 10 SARS-CoV-2 recovered-vaccinated donors that exhibited cross-reactive binding to this spike region.

\section{Isolation of a large panel of $\beta$-CoV spike stem-helix mAbs}

Using SARS-CoV-2 and MERS-CoV S-proteins as baits, we sorted antigen-specific single $B$ cells to isolate 40 stem-helix mAbs from 10 COVID-19 convalescent donors who had been recently vaccinated with the Pfizer/BioNTech BNT162b2 $(n=4:$ CC9, CC92, CC95 and CC99), Johnson \& Johnson Ad26.CoV2.S ( $n=1$ : CC67), or Moderna mRNA-1273 ( $\mathrm{n}=5$ : CC24, CC25, CC26, CC67, CC84) vaccines (Fig. 1c, d, Supplementary Fig. 1) (2, 58, 59). Briefly, using SARS-CoV-2 and MERS-CoV S-proteins, we sorted $\mathrm{CD}_{19}{ }^{+} \mathrm{CD} 20^{+} \operatorname{lgG} \mathrm{IgM}^{-} \mathrm{B}$ cells positive for both probes from the peripheral blood mononuclear cells (PBMCs) of these donors. Flow cytometry profiling revealed up to $36 \%$ (range $=6-36 \%$, median $=15 \%$ ) SARS-CoV-2 S-protein-specific B cells, of which a sizable fraction was cross-reactive with the MERS-CoV S-protein (range $=0.04-0.28 \%$, median $=0.16 \%$ total selected B cells) (Supplementary Fig. $1 \mathrm{~b}$ ). A total of 358 SARSCoV-2: MERS-CoV S-protein-specific double positive single B cells were recovered from the 10 donors, of which the heavy (HC)-light (LC) chain pairs were recovered from 247 single B cells (69\%) from 9 donors and expressed as lgGs (Supplementary Fig. 1C). Expi293F cell-expressed IgG supernatants of $247 \mathrm{mAbs}$ were screened for dual binding to SARS-CoV-2 and MERS-CoV stem-helix peptides and 16\% (40/247) exhibited crossreactive binding (Supplementary Fig. 1c). Dual binding was confirmed for the corresponding purified IgGs. Except for two mAbs that failed to bind HCoV-HKU1 stemhelix peptide, all mAbs exhibited cross-reactive binding to stem-helix peptides of endemic $\beta$-HCoV (HCoV-HKU1 and HCoV-OC43) but not $\alpha-H C o V$ (HCoV-NL63 and HCoV-229E) (Fig. 1c). We also tested binding of mAbs to soluble HCoV S-proteins and cell surface expressed spikes and observed consistent binding to SARS-CoV-2/1 and MERS-CoV spikes but reduced binding to endemic $\beta$-HCoV spikes (HCoV-HKU1 and HCoV-OC43), especially in the soluble S-protein format (Supplementary Fig. 2). Overall, we isolated 40 stem-helix mAbs, of which 32 were encoded by unique immunoglobulin germline gene combinations and 7 were expanded lineages with 2 or more clonal members (Fig. 1c, Supplementary Fig. 2). 


\section{Spike stem-helix mAbs exhibit broad neutralization against $\beta$-CoVs}

We next examined neutralization of stem-helix mAbs against clade 1a (SARS-CoV-1, WIV1 and SHC014) and clade 1b (SARS-CoV-2 and Pang17) ACE2-utilizing sarbecoviruses $(26,27)$ and MERS-CoV (28). Consistent with conservation of the stemhelix bnAb epitope region across sarbecoviruses, all the $32 \mathrm{mAb}$ lineages neutralized all the 5 sarbecoviruses tested with widely varying degrees of neutralization potency (Fig. 1c, d). The bnAbs neutralized clade 1a SHC014 and clade 1b SARS-CoV-2 relatively more potently compared to the other sarbecoviruses, but some bnAbs neutralized all viruses in the lower $\mu \mathrm{g} / \mathrm{mL} \mathrm{IC}_{50}$ neutralization titer range $(0.1$ to $3 \mu \mathrm{g} / \mathrm{ml})$. Of 32 unique stem-helix bnAb lineages, 23 (72\%) bnAbs neutralized MERS-CoV (Fig. 1c, d). Neutralization potency against MERS-CoV was lower compared to the sarbecoviruses but many bnAb members were consistently effective. We tested neutralization of SARSCoV-2 VOCs (B.1.1.7 (Alpha), B.1.351 (Beta), P.1 (Gamma), B.1.617.2 (Delta) and B.1.1.529 (Omicron) by select bnAbs (Fig. 2a). Consistent with the conservation of the stem-helix region in SARS-CoV-2 VOCs, these bnAbs were consistently effective against the VOCs tested (Fig. 2a). Of note, a fraction of stem-helix bnAbs showed some degree of polyreactivity or autoreactivity in HEp2 cell or polyspecificity reagent (PSR) assays ( 7 ) but the majority were negative (Supplementary Figs. 2 \& 4). Overall, we have identified multiple stem-helix bnAbs that exhibit broad neutralizing activity against phylogenetically diverse $\beta$-HCoVs.

\section{Immunogenetics of stem-helix bnAbs and vaccine targeting}

Immunogenetic analysis of stem-helix antibody sequences showed strong enrichment of IGHV1-46 (63\%) and IGHV3-23 (22\%) germline gene families as compared to human baseline germline frequencies (Figs. 1c, 2b, Supplementary Fig. 3) (60, 61). Of note, previously isolated stem-helix human bnAbs, S2P6 and CC40.8, are IGHV1-46 and IGHV3-23 germline encoded, respectively $(44,45)$. The IGHV1-46 germline gene was slightly more enriched (78\%) in stem-helix bnAbs that exhibited MERS-CoV neutralization in addition to sarbecoviruses, suggesting a potential role for this $\mathrm{VH}$-germline gene for broader reactivity against diverse $\beta$-HCoV spikes. Interestingly, at least one IGHV1-46encoded stem-helix bnAb was isolated from each of the 9 donors and may represent a public clonotype for this bnAb site. For light chain gene usage, we noted a strong enrichment of IGKV3-20 (47\%) and to some degree IGLV1-51 (16\%) germline gene families as compared to human baseline germline frequencies (Figs. 1c, 2c, Supplementary Fig. 3) (62). The mAbs possessed modest levels of V-gene nucleotide somatic hypermutation (SHM): for $\mathrm{VH}$, median $=7.3 \%$ and for $\mathrm{VL}$, median $=4.5 \%$ (fig. S2).

We examined the CDRH3 loop lengths in the isolated stem-helix bnAbs and observed a strong enrichment for 10- and 11-residue long CDRH3s compared to the human baseline reference database (Fig. 2d, Supplementary Fig. 3) (60, 61). No apparent enrichment in germline D-genes was observed but IGHJ4, the most common germline J-gene utilized in humans, was slightly enriched (72\%) in stem-helix bnAbs compared to a reference germline database (Supplementary Fig. 3) $(60,61)$. We also examined the CDRL3 loop lengths in the stem-helix bnAbs and observed strong enrichment for 9- and 11-residue 
CDRL3s (Fig. 2e, Supplementary Figs. 2 and 3). These CDRL3 loops possess germline JL-gene-encoded motifs (Fig. 2f, Supplementary Fig. 2), which may be important for epitope recognition. Overall, we observed a strong enrichment of IGHV and IGLV germline gene features in $\beta$-HCoV spike stem-helix bnAbs. Therefore, rational vaccine strategies may exploit these germline gene features to generate a protective $B$ cell response $(53,54,63)$.

To examine the potential contribution of antibody SHMs to SARS-CoV-2 neutralization efficiency and cross-neutralization with MERS-CoV, we tested the binding of select mAbs (based on a broad range of neutralization potency) to SARS-CoV-2 or MERS-CoV monomeric stem-helix peptides and to their S-proteins by BLI (Supplementary Fig. 5). The mAbs bind SARS-CoV-2 and MERS-CoV-2 stem-helix peptides with nanomolar (nM) and higher $K_{D}$ affinity (Supplementary Fig. 5a) and were generally higher for SARS-CoV2 compared to MERS-CoV stem-helix peptide. We found no association of heavy or light chain SHMs with binding to SARS-CoV-2 or MERS-CoV-2 stem-helix peptides or with neutralization of the corresponding viruses (Supplementary Fig. 5b). We however observed a strong association of binding affinity to stem-helix peptides and neutralization (Supplementary Fig. 5c).

To further investigate the role of SHM in binding and neutralization, we generated inferred germline (iGL) versions of stem-nAbs by reverting their heavy and light chain V, D and J regions to the corresponding germlines (inferred germlines, iGLs) as described previously (64) and assessed both binding and neutralization. The BLI binding responses and the $\mathrm{K}_{\mathrm{D}}$ values of the bnAb iGLs with SARS-CoV-2 and MERS-CoV stem-helix peptides were substantially reduced compared to mature bnAbs but were still strong and in the lower $\mathrm{nM}$ and higher $\mathrm{K}_{\mathrm{D}}$ affinity range (Fig. 3a, Supplementary Fig. 5a). We observed higher affinities or CDRH3 "RG" motif-bearing IGHV1-46-encoded and CDRL3 "WD" motifbearing IGLV1-51-encoded bnAb iGLs for binding to SARS-CoV-2 or MERS-CoV stemhelix peptides (Fig. 3a, Supplementary Fig. 6). Binding of bnAbs and their iGLs to Sproteins were generally of higher affinity than to the corresponding peptides, possibly due to avidity effects (Supplementary Fig. 5a). The affinities of iGLs compared to mature bnAbs were notably less for S-proteins compared to the corresponding peptides, particularly for the MERS-CoV S-protein where many of the iGL Abs failed to bind substantially (Supplementary Fig. 5a). Overall, these results suggest a significant contribution from germline-encoded residues to epitope binding, in most cases consistent with enrichment of certain antibody germline gene features above (Figs. 1 and 2).

In contrast to binding, neutralization of SARS-CoV-2 and MERS-CoV by stem-helix bnAb iGLs was absent (Supplementary Fig. 5d). The result suggests that, although overall SHM levels do not correlate with binding or neutralization, key antibody mutations are critical for the neutralization phenotype to attain sufficient affinity for neutralization to be observed.

Altogether, we have isolated a large panel of human $\beta$-CoV bnAbs that are enriched in certain germline gene features suggesting the potential value of a highly targeted 
approach $(53,54,63)$ to induce pan-betacoronavirus bnAbs by vaccines in which the immunogen and vaccination strategies are appropriately designed.

\section{Spike stem-helix bnAbs recognize a common hydrophobic core epitope}

To determine the epitope specificities of the isolated stem-helix bnAbs and potential association with antibody immunogenetic properties, we performed binding of all 32 stem bnAbs to alanine scanning mutants of the SARS-CoV-2 stem-peptide (Fig. 3c, Supplementary Fig. 7). A dependence on three hydrophobic residues, $F^{1148}$, $L^{1152}$, and $\mathrm{F}^{1156}$, by many bnAbs that form a common core epitope was identified but the relative dependence of bnAb lineages on each of the hydrophobic core residues varied. Many of the IGHV1-46-encoded bnAbs were paired with IGVK3-20 or IGLV1-51 light chain and all except two bnAbs possessed a CDRL3 of 11 residues. The IGHV3-23-encoded bnAbs showed dependence on 1 or 2 hydrophobic core epitope residue and some lineages showed dependence on an upstream acidic residue, $\mathrm{D}^{1146}$. All of the IGHV3-23 encoded bnAbs were paired with a IGVK3-20 light chain with a 9-residue long CDRL3 loop. The non -IGHV1-46 or -IGHV3-23-encoded stem-helix bnAbs were also dependent on one or more hydrophobic core epitope residues with one exception. Structural analysis of the IGHV1-46-encoded S2P6 or IGHV3-23-encoded CC40.8 stem-helix bnAbs shows that antibody germline gene-encoded residues are involved in recognition of the hydrophobic bnAb epitope (Fig. 3d-i). Overall, hydrophobic core residues in the spike fusion machinery, which are highly conserved across betacoronaviruses, are important targets for S2 bnAbs. Notably, the hydrophobic core epitope residues on the pre-fusion S-trimer are poorly accessible and partial disruption of the stem-helix region may be needed to favorably expose this bnAb site to engage desired $B$ cell responses $(43-45,48)$.

\section{Stem-helix bnAbs protect against challenge with diverse $\beta$-CoVs}

To determine the protective efficacy of the stem-helix bnAbs, we prophylactically treated aged mice (65) with individual antibodies followed by virus challenge. We selected two of the broadest and potent stem-helix bnAbs, CC68.109 and CC99.103, and investigated their in vivo protective efficacy against all three major human disease-causing betacoronaviruses; SARS-CoV-2, SARS-CoV-1 and MERS-CoV. Prior to the challenge experiments, we examined neutralization of SARS-CoV-2 and MERS-CoV replicationcompetent viruses by the two candidate bnAbs and compared with that of pseudoviruses (Supplementary Fig. 8). The neutralization $I_{50 S}$ of the stem-helix bnAbs were comparable for SARS-CoV-2 across the two assay formats while the titers with replication-competent MERS-CoV were more effective (lower $\mathrm{IC}_{50}$ values) compared to the pseudovirus format. The two stem-helix bnAbs, individually, or a DEN3 control antibody were administered intra-peritoneally (i.p.) at $300 \mu \mathrm{g} / \mathrm{animal}$ into 9 groups of 10 animals ( 3 groups per antibody; Fig. 4a). 12h prior to the virus challenge, the test antibody in each animal group was administered followed by intranasal (i.n.) challenge with one of three mouse-adapted (MA) betacoronaviruses, (MA10-SARS-2 = SARS-CoV-2; MA15SARS- 1 = SARS-CoV-1 or M35c4-MERS = MERS-CoV) (Fig. 4a) (65-67). Post virus challenge, the animals were monitored for signs of clinical disease due to infection, including daily weight changes, and pulmonary function. Animals were euthanized at day 2 or day 4 post infection and lung tissues were harvested to assess gross pathology. Compared to the control antibody DEN3-treated animal groups, the stem-helix bnAb- 
treated animals in all three betacoronaviruses challenge experiments showed substantially reduced weight loss (Fig. 4b, e, h), reduced hemorrhage (Fig. 4c, f, i), and normal pulmonary function (Fig. $4 \mathrm{~d}, \mathrm{~g}$, j), suggesting a protective role for the bnAbs.

Overall, both stem-helix bnAbs protected against severe betacoronavirus disease, CC99.103 being slightly more protective than CC68.109 bnAb.

\section{Discussion}

In terms of passive antibody treatment, the ability of single mAbs to protect against the two SARS viruses and MERS in the small animal model is encouraging for their potential adoption as stockpiled reagents to tackle future outbreaks of viral infection, including novel related betacoronaviruses. Prophylaxis or treatment very early in infection is more likely to be successful than therapy once symptoms are established, based on experience with SARS-CoV-2 $(68,69)$. The use of S2 bnAbs, possibly in a cocktail with the most appropriate RBD bnAbs, may be the optimal approach for SARS-CoV-2 prophylaxis, especially as new variants such as Omicron emerge. The dose of S2 bnAbs required to be effective, given the typically lower neutralization potencies of such nAbs compared to RBD nAbs, may be an issue for translation. However, the studies in animal models (44, 45 ) suggest that $\mathrm{S} 2$ bnAbs protect at much lower serum concentrations than would be predicted by their $\mathrm{IC}_{50}$ neutralizing titers-i.e. they "punch above their weight". Therefore, in addition to neutralization, effector functions of the S2 bnAbs may be important for protection $(45,70-72)$ and clinical studies will be required to investigate this phenomenon in humans.

300

301

302

In terms of vaccine design, a rational strategy is strongly favored by the availability of a panel of bnAbs rather than single mAbs so that the broadly neutralizing epitope can be more precisely defined and the qualities of nAbs required for broad neutralization determined $(53,54,56,63,73,74)$. Here, we identified critical hydrophobic $\$ 2$ residues involved in bnAb binding and showed the prevalence of a IGVH1-46/IGVK3-20 antibody pairing with restricted $\mathrm{CDRH} 3$ and CDRL3 lengths in S2 bnAbs. Accordingly, rational vaccine design strategies may take advantage of these germline gene features to develop immunogens that can induce protective antibody responses to this site $(52-54,56,63)$. Accessibility of the $\mathrm{S} 2$ stem-helix bnAb site on spike immunogen to effectively engage desired $B$ cell responses might be challenging. Nevertheless, approaches to scaffold immunogen designs that can accommodate these features are now available to be deployed $(46,52,56,75-77)$.

In summary, we isolated the largest panel of $\beta$-CoV bnAbs to date and revealed the molecular basis for their broad protection. The bnAbs provide a detailed framework for rational design of broad coronavirus vaccines and themselves could be used as reagents to counter betacoronavirus spillovers. 
Figures and Legends

323

324 a

Sarbecovirus Merbecovirus

Embecovirus Alpha-coronavirus

COVID-19 sera
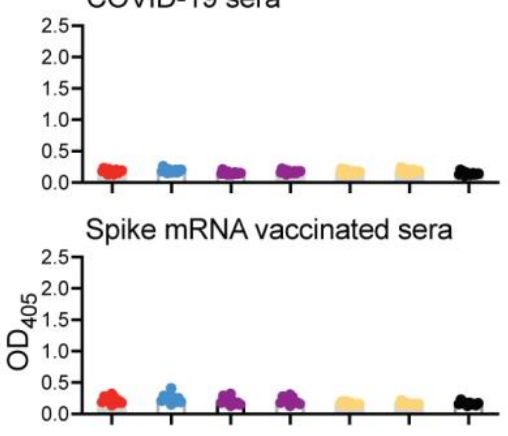

Recovered vaccinated sera
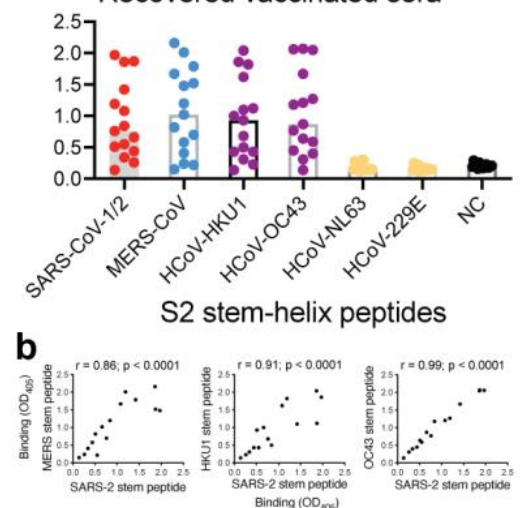
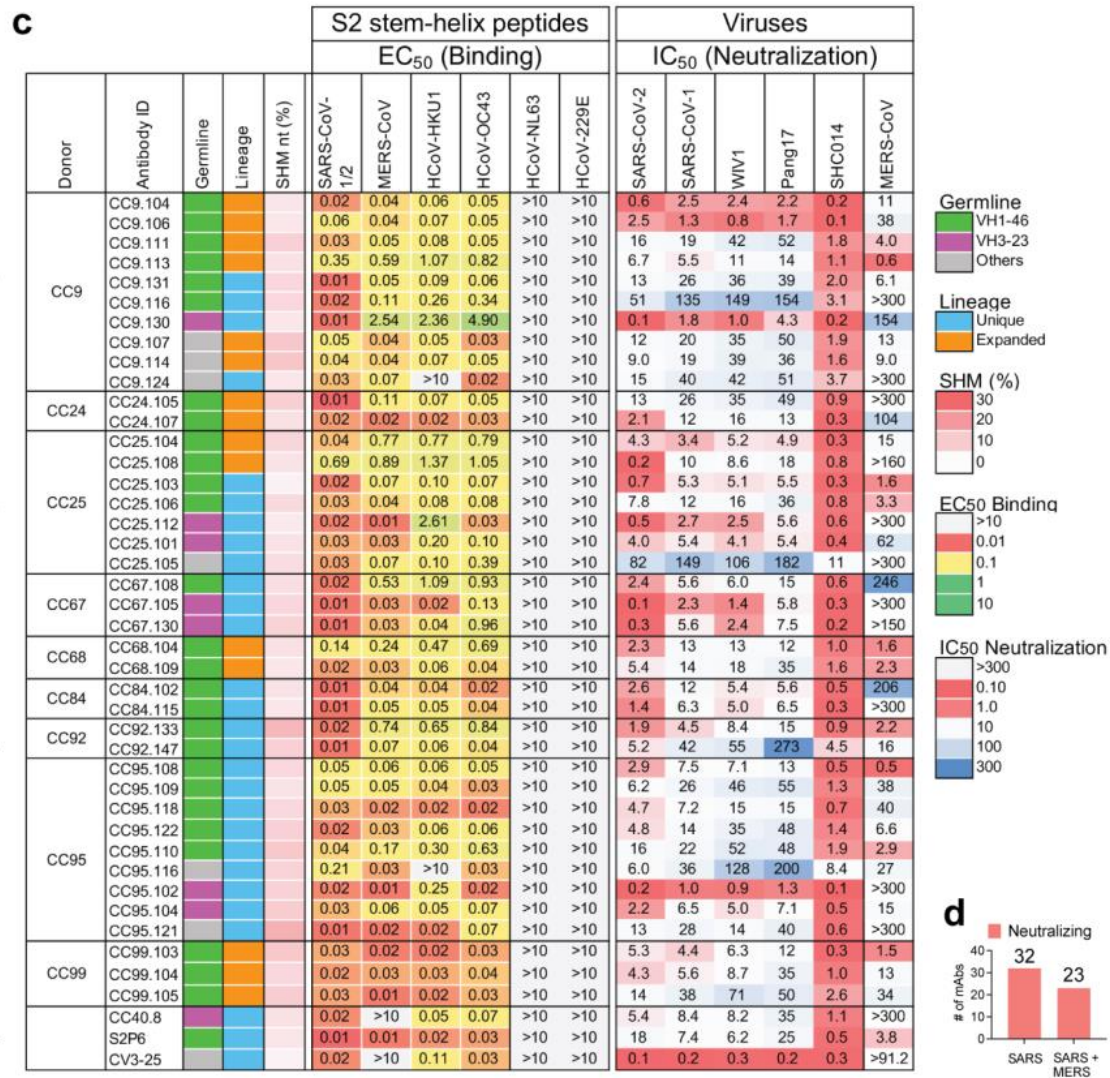

Figure. 1. Binding and neutralization properties of S2 stem-helix mAbs. a. Dot plots showing ELISA binding (OD 405$)$ reactivity of immune sera from COVID-19 convalescent donors $(n=15)$, spike mRNA-vaccinated donors $(n=10)$ and SARS-CoV-2 recoveredvaccinated donors $(n=15)$ to 25 -mer peptides corresponding to spike S2 stem-helix regions of human $\beta$-(sarbecoviruses: SARS-CoV-1 or 2; merbecovirus: MERS-CoV; embecoviruses: HCoV-HKU1, HCoV-OC43) and $\alpha-(H C o V-N L 63$ and HCoV-229E) coronaviruses. 12 out of 15 (80\%) SARS-CoV-2 recovered-vaccinated donor sera show cross-reactive binding to $\beta$-CoV spike stem-helix peptides. b. Correlation between binding of infected-vaccinated sera to SARS-CoV-2 stem-helix peptide and the other $\beta$-CoV (MERS-CoV, HCoV-HKU1 and HCoV-OC43) stem-helix peptides. Responses for binding to two stem-helix peptides were compared by nonparametric Spearman correlation twotailed test with $95 \%$ confidence interval and the Spearman correlation coefficient $(r)$ and the $p$-value are indicated. c. A total of $40 \mathrm{~S} 2 \mathrm{stem}$-helix mAbs were isolated from 9 SARSCoV-2 recovered-vaccinated donors (CC9, CC24, CC25, CC67, CC68, CC84, CC92, CC95 and CC99). MAbs were isolated by single B cell sorting using SARS-CoV-2 and MERS-CoV S-proteins as baits. Heatmap showing IGVH germline gene usage (colored: VH1-46 (green), VH3-23 (plum) and other V-genes (grey)), lineage information (unique (sky) and expanded (tangerine) lineages) and V-gene nucleotide somatic hypermutations (SHMs). EC 50 ELISA binding titers of mAbs with $\beta$ - and $\alpha$-HCoV spike S2 stem-helix region peptides. MAbs showed binding to $\beta$ - but not $\alpha-\mathrm{HCoV}$ derived stem-helix peptides. IC 50 neutralization of mAbs against pseudoviruses of clade1a (SARS-CoV-2 and Pang17), clade 1b (SARS-CoV-1, WIV1, SHC014) sarbecoviruses and MERS-CoV. 
bioRxiv preprint doi: https://doi.org/10.1101/2022.03.04.479488; this version posted March 7, 2022. The copyright holder for this preprint (which was not certified by peer review) is the author/funder, who has granted bioRxiv a license to display the preprint in perpetuity. It is made available under aCC-BY-NC-ND 4.0 International license.

347 Spike S2 stem-helix bnAbs, CC40.8, S2P6 and CV3-25 were used as controls for binding 348 and neutralization assays. d. 32 of 40 stem-helix bnAbs were unique clones that 349 neutralized all ACE2-utilizing sarbecoviruses and 23 out of 32 unique mAb neutralized 350 MERS-CoV, in addition to sarbecoviruses. 
bioRxiv preprint doi: https://doi.org/10.1101/2022.03.04.479488; this version posted March 7, 2022. The copyright holder for this preprint (which was not certified by peer review) is the author/funder, who has granted bioRxiv a license to display the preprint in perpetuity. It is made available under aCC-BY-NC-ND 4.0 International license.
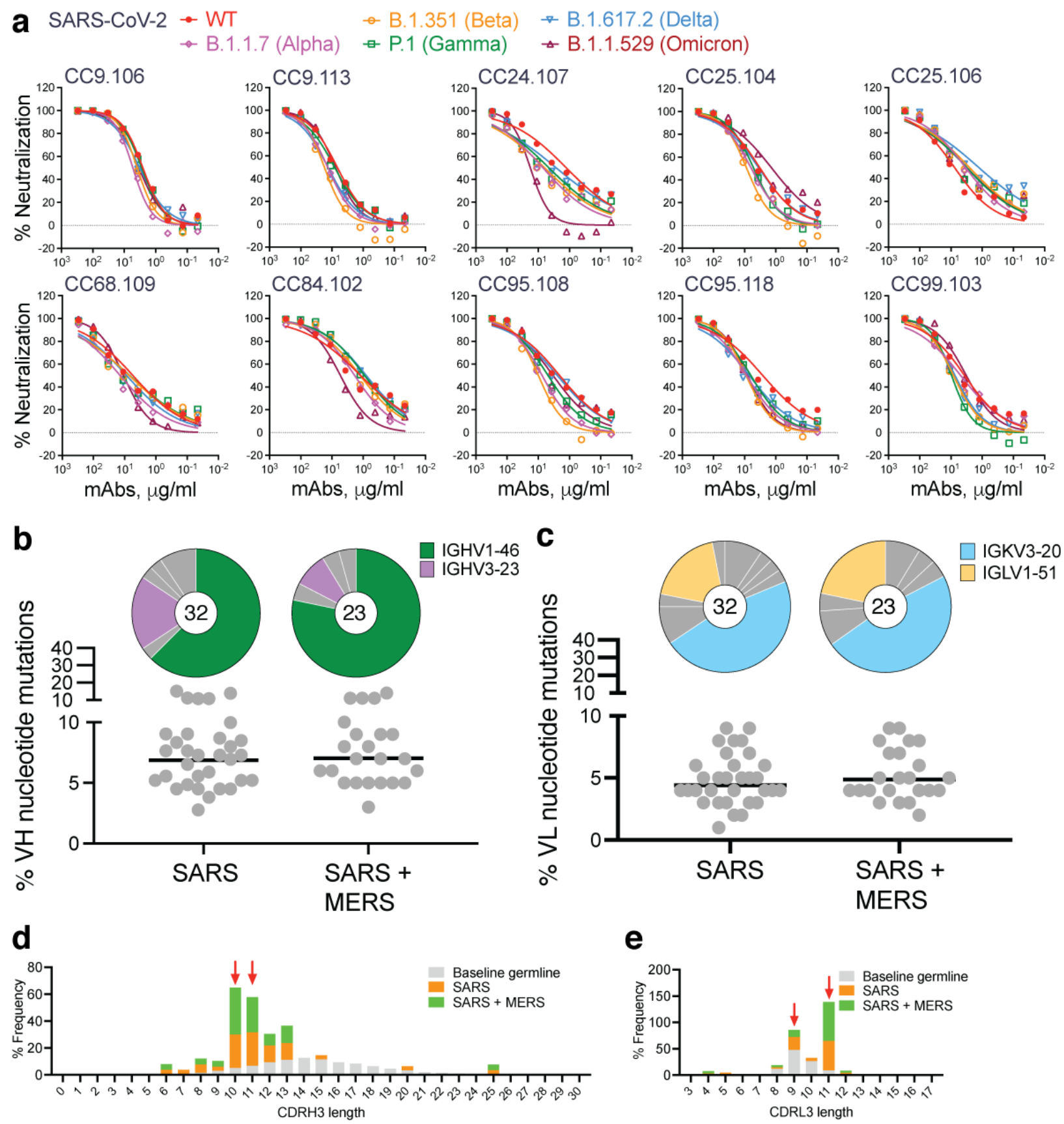

f $9 \operatorname{AACDRL3s}(n=8)$
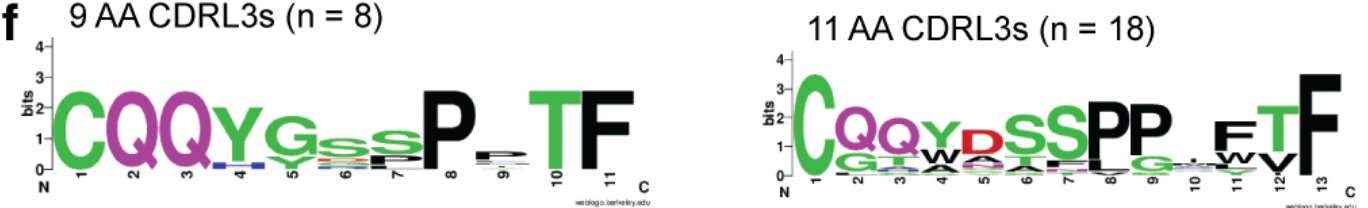

Figure. 2. Neutralization of SARS-CoV-2 VOCs, and immunogenetic properties of S2 $\beta$-CoV spike stem-helix bnAbs. a. Neutralization of 10 select S2 stem-helix bnAbs against SARS-CoV-2 (WT) and five major SARS-CoV-2 variants of concern [B.1.1.7 (Alpha), B.1.351 (Beta), P.1 (Gamma), B.1.617.2 (Delta), and B.1.1.529 (Omicron)]. b-c. Pie plots showing IGHV and IGKV/IGLV gene usage distribution of isolated stem-helix mAbs. Enriched heavy (IGHV1-46 (green) and IGHV3-23 (plum)) (b) and light (IGKV3-20 
359 (sky) and IGLV1-51 (cantaloupe)) (c) gene families are colored. Dot plots showing \% 360 nucleotide mutations (SHMs) in the heavy (VH) or light ( $\mathrm{VL}$ ) chains of isolated stem-helix $361 \mathrm{mAbs}$. The mAbs are grouped by neutralization against sarbecoviruses or sarbecoviruses 362 + MERS-CoV. d-e. CDRH3 (d) or CDRL3 (e) length distributions of isolated mAbs across 363 sarbecovirus broadly neutralizing and sarbecovirus + MERS-CoV broadly neutralizing 364 mAb groups compared to human baseline germline reference. MAbs with 10- and 11365 amino acid-CDRH3s or mAbs with 9- and 11- amino CDRL3s, enriched in S2 stem-helix 366 bnAbs compared to baseline germline reference, are indicated by red arrows. $f$. 367 Sequence conservation logos of $9(n=8)$ and $11(n=18)$ amino acid long CDRL3-bearing 368 stem-helix bnAbs show enrichment of certain J-gene encoded residues. 
bioRxiv preprint doi: https://doi.org/10.1101/2022.03.04.479488; this version posted March 7, 2022. The copyright holder for this preprint (which was not certified by peer review) is the author/funder, who has granted bioRxiv a license to display the preprint in perpetuity. It is made available under aCC-BY-NC-ND 4.0 International license.
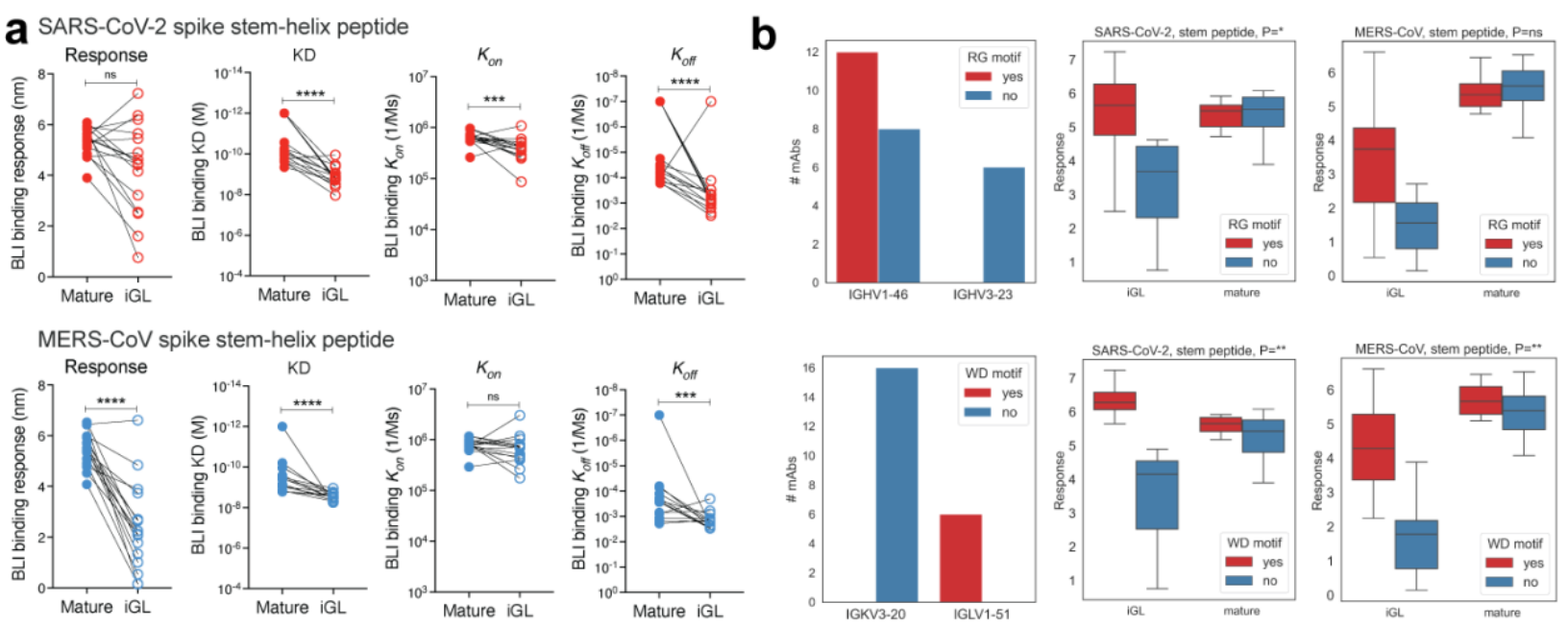

C

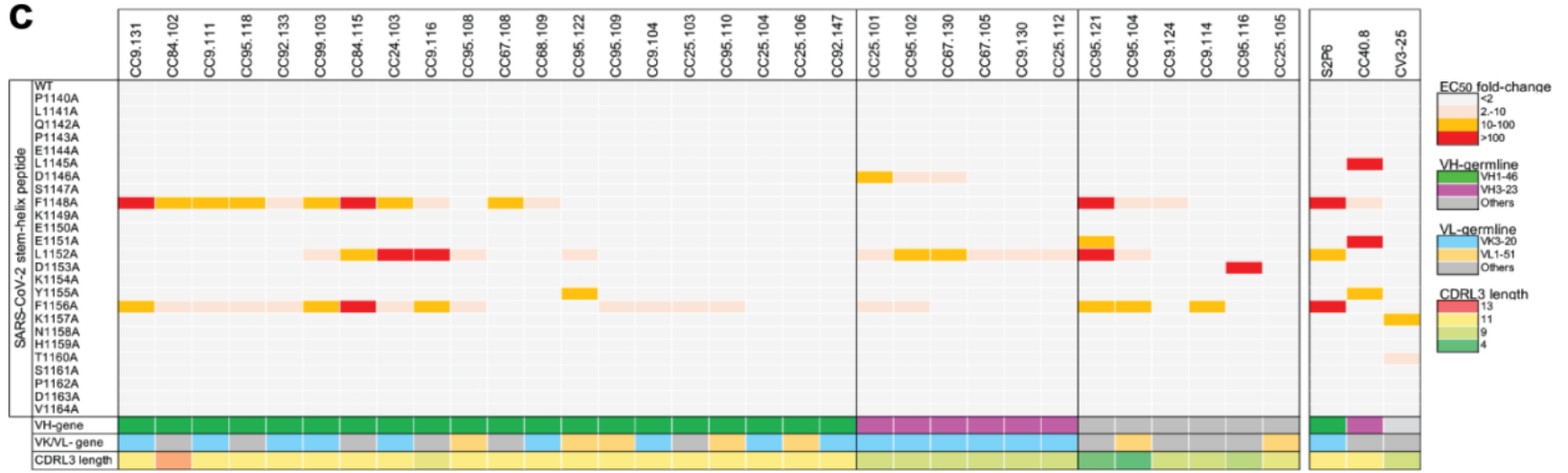

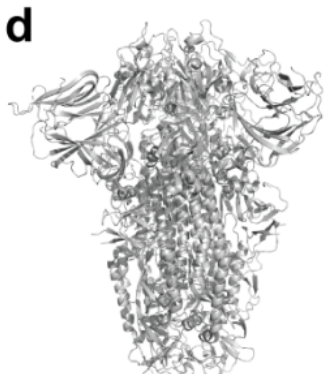

Stem-helix

Stem-helix
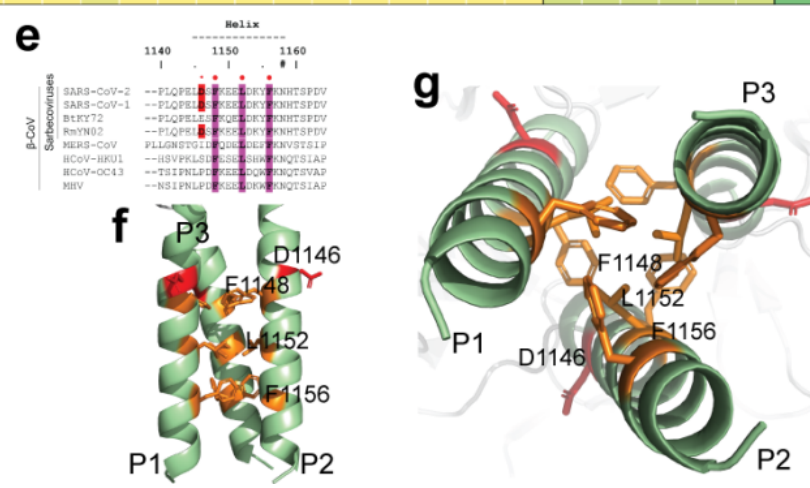

h \$2P6

i $\mathrm{CC} 40.8$
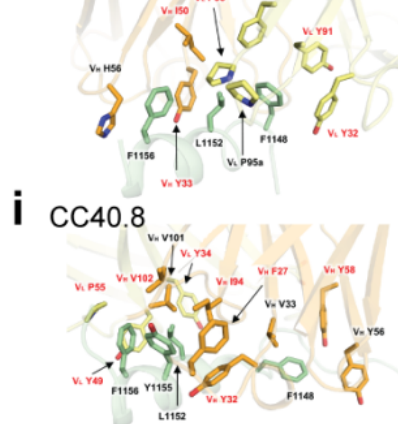

Figure. 3. Binding kinetics and fine epitope specificities of S2 $\beta-\mathrm{CoV}$ spike stemhelix bnAbs. a. BLI binding kinetics of $S 2$ stem-helix mature bnAbs and their inferred germline (iGL) versions to monomeric SARS-CoV-2 and MERS-CoV stem-helix peptides. Binding kinetics were obtained using a 1:1 binding kinetics fitting model on ForteBio Data Analysis software and maximum binding responses, dissociations constants $\left(K_{D}\right)$ and onrate $\left(k_{o n}\right)$ and off-rate constants $\left(k_{\text {off }}\right)$ for each antibody-protein interaction are compared. $K_{D}$, $K_{o n}$ and $k_{\text {off }}$ values were calculated only for antibody-antigen interactions where a maximum binding response of $0.2 \mathrm{~nm}$ was obtained. The S2 stem-helix bnAb iGL Abs showed substantially reduced binding to stem-helix peptides compared to their corresponding mature versions. Statistical comparisons between two groups were performed using a Mann-Whitney two-tailed test, ${ }^{* * *} p<0.001$, ${ }^{* * *} p<0.0001$; ns- $p$ $>0.05)$. b. Association of $S 2$ stem-helix peptide binding by $S 2$ bnAbs and their iGLs with 
CDRH3 and CDRL3 motifs. The top line shows characteristics of mAbs with (red) and without (blue) RG motifs in CDRH3s. The bottom line shows characteristics of mAbs with (red) and without (blue) WD motifs in CDRL3s. The left column shows the numbers of mAbs with and without CDR3 motifs with respect to two most common V genes: IGHV146 and IGHV3-23 for RG motifs in CDRH3, IGKV3-20 and IGLV1-51 for WD motifs in CDRL3s. The middle and right columns show the responses of $\mathrm{iGL}$ and mature $\mathrm{mAbs}$ to stem peptides of SARS-CoV-2 and MERS-CoV, respectively. P-values of associations between RG / WD motifs and the responses are shown on tops of the plots and denoted as follows: ns $\geq 0.05,{ }^{*}<0.05,{ }^{* *}<0.005$. P-values are computed using linear regression. $\mathbf{c}$. ELISA-based epitope mapping of S2 stem-helix bnAbs with SARS-CoV-2 stem alanine scan peptides (25mer). Heatmap shows fold-changes in $\mathrm{EC}_{50}$ binding titers of mAb binding to SARS-CoV-2 stem-helix peptide alanine mutants compared with the WT peptide. SARS-CoV-2 stem-helix residue positions targeted (2-fold or higher decrease in $\mathrm{EC}_{50}$ binding titer compared to WT stem peptide) is indicated in colors. Three hydrophobic residues, $\mathrm{F}^{1148}$, $\mathrm{L}^{1152}$ and $\mathrm{F}^{1156}$, were commonly targeted by stem-helix bnAbs and that form the core of the bnAb epitope. Association of dependence on the stem bnAb core epitope residues with heavy (IGHV1-46 and IGHV3-23) and light (IGKV3-20 and IGLV151) chain genes usage and CDRL3 lengths is shown. d-g. A SARS-CoV-2 spike protein cartoon depicts the S2-stem epitope region in green at the base of the prefusion spike ectodomain (d). Sequence conservation of stem-helix hydrophobic core epitope residues $\left(F^{1148}, L^{1152}\right.$ and $F^{1156}$ ) across $\beta$-coronavirus spikes (PDB: 6XR8) (e). $D^{1146}$ stem-helix residue is also indicated. Side (f) and top ( $g$ ) views of spike stem-helix region highlight the core epitope residues. h.i. Interactions between SARS-CoV-2 S2 stem helix with (h) S2P6 and (i) CC40.8 highlighting the contribution of antibody germline-encoded residues in recognition of hydrophobic stem-helix core epitope. The SARS-CoV-2 S2 stem helices are shown in green, while heavy and light chains of antibodies in orange and yellow, respectively. Germline gene encoded residues are highlighted in red. Structures with PDB codes 7RNJ and 7SJS are used for S2P6 and CC40.8, respectively. 
a
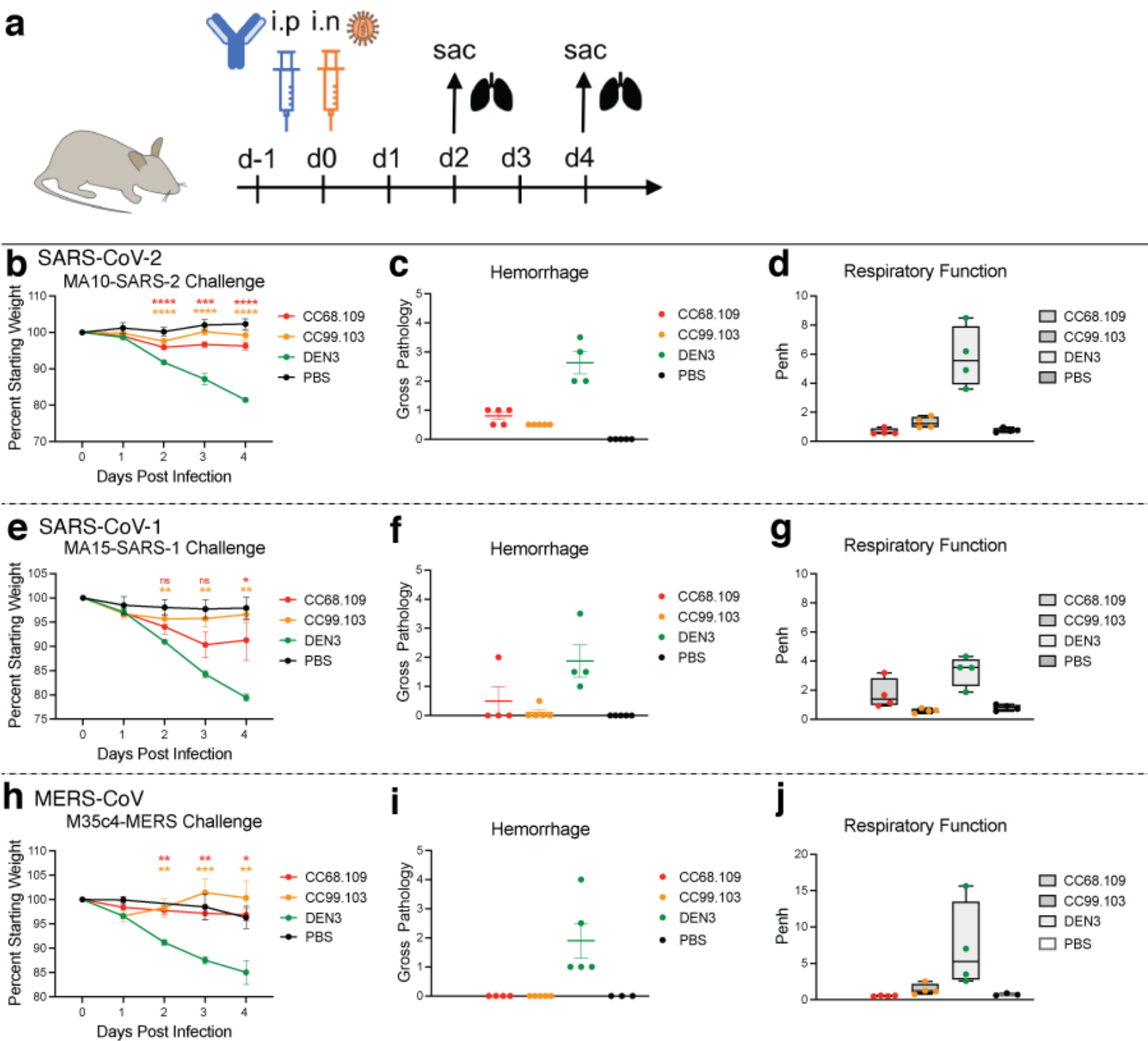

Figure. 4. Prophylactic treatment of aged mice with $\mathrm{S} 2$ stem-helix bnAbs protects against challenge with diverse betacoronaviruses. a. Two $\mathrm{S} 2$ stem-helix bnAbs (CC68.109 and CC99.103) individually, or a DEN3 control antibody were administered intra-peritoneally (i.p.) at $300 \mu \mathrm{g}$ per animal into 9 groups of aged mice (10 animals per group). Each group of animals was challenged intra-nasally (i.n.) $12 \mathrm{~h}$ after antibody infusion with one of 3 mouse-adapted (MA) betacoronaviruses, (MA10-SARS-2 = SARSCoV-2; $1 \times 10^{3}$ plaque forming units (PFU), MA15-SARS1 = SARS-CoV-1; $1 \times 10^{5}$ PFU or M35c4-MERS = MERS-CoV; $\left.1 \times 10^{3} \mathrm{PFU}\right)$. As a control, groups of mice were exposed only to PBS in the absence of virus. b., e., h. Percent weight change in S2 stem-helix bnAbs or DEN3 control antibody-treated animals after challenge with mouse-adapted betacoronaviruses. Percent weight change was calculated from day 0 starting weight for all animals. c., f., i. Day 2 post-infection Hemorrhage (Gross Pathology score) scored at tissue harvest in mice prophylactically treated with S2 stem-helix bnAbs or DEN3 control mAb. d., g., j. Day 2 post-infection pulmonary function (shown as Penh score) was measured by whole body plethysmography in mice prophylactically treated with S2 stem- 
bioRxiv preprint doi: https://doi.org/10.1101/2022.03.04.479488; this version posted March 7, 2022. The copyright holder for this preprint (which was not certified by peer review) is the author/funder, who has granted bioRxiv a license to display the preprint in perpetuity. It is made available under aCC-BY-NC-ND 4.0 International license.

428 helix bnAbs or DEN3 control mAb. Statistical comparisons between groups were 429 performed using a Kruskal-Wallis non-parametric test and significance was calculated 430 with Dunnett's multiple comparisons test between each experimental group and the DEN3 control Ab group. $\left(p<0.05,{ }^{* *} p<0.01,{ }^{* *} p<0.001 ;{ }^{* * *} p<0.0001\right.$; ns- $\left.p>0.05\right)$. 
434

435

436

437

438

439

440

441

442

443

444

445

446

447

448

449

450

451

452

453

454

455

456

457

458

459

460

461

462

463

464

465

466

467

468

469

470

471

\section{Author contributions}

P.Z., G.S., R.S.B., L.E.G., D.R.B. and R.A. conceived and designed the study. N.B., M.P., E.G., S.A.R., D.M.S., and T.F.R. recruited donors and collected and processed plasma and PBMC samples. P.Z., G.S., W.H., R.M., K.D., S.C., P.Y., L.P., T.C., O.L. and F.A. performed BLI, ELISA, virus preparation, neutralization and isolation and characterization of monoclonal antibodies. Y.S. performed immunogenetic analysis of antibodies. M.Y. and H.L. generated antibody-antigen structural models. L.V.T. performed live virus neutralizations assays and D.R.M., A.S., and L.E.G. conducted in vivo animal protection studies. P.Z., G.S., W.H., N.B., L.V.T., D.R.M., A.S., F.A., P.Y., L.P., K.D., R.M., S.C., T.C., M.Y., H.L., O.L., M.P., E.G., D.N., J.G.J., I.A.W., Y.S., T.F.R., R.S.B., L.E.G., D.R.B. and R.A. designed the experiments and/or analyzed the data. P.Z., G.S., D.R.B. and R.A. wrote the paper, and all authors reviewed and edited the paper.

\section{Acknowledgements}

We thank all the human cohort participants for donating samples. This work was supported by NIH CHAVD UM1 Al44462 (D.R.B.), NIH R61 Al161818 (R.A.), the IAVI Neutralizing Antibody Center, the Bill and Melinda Gates Foundation INV-004923 (I.A.W., D.R.B.), the Translational Virology Core of the San Diego Center for AIDS Research (CFAR) grant NIH Al036214 (D.M.S.), NIH 5T32AI007384 (S.A.R.), NIH U54 CA260543 and Al157155 (R.S.B), NIH R21 Al145372 (L.E.G.), and the John and Mary Tu Foundation and the James B. Pendleton Charitable Trust (D.M.S. and D.R.B.). L.V.T. is supported by Pfizer NCBiotech Distinguished Postdoctoral Fellowship in Gene Therapy. D.R.M. is currently supported by a Burroughs Wellcome Fund Postdoctoral Enrichment Program Award and a Hanna H. Gray Fellowship from the Howard Hugues Medical Institute.

\section{Competing interests}

P.Z., G.S., W.H., D.R.B. and R.A. are listed as inventors on pending patent applications describing the betacoronavirus broadly neutralizing antibodies isolated in this study. D.R.B. is a consultant for IAVI and for Adagio. RSB and LEG have ongoing collaborations with Adagio. All other authors have no competing interests to declare.

Data availability: The data supporting the findings of this study are available within the paper and its supplementary information files or from the corresponding author upon reasonable request. Antibody sequences have been deposited in GenBank under accession numbers XXXX-XXXX. Antibody plasmids are available from Raiees Andrabi or Dennis Burton under an MTA from The Scripps Research Institute. 
472

473

474

475

476

477

478

479

480

481

482

483

484

485

486

487

488

489

490

491

492

493

494

495

496

497

498

499

500

501

502

503

504

505

506

507

508

509

510

511

512

513

514

515

516

517

\section{Methods}

\section{COVID-19 infected-vaccinated donors}

Sera and PBMC samples from convalescent COVID-19 donors, vaccinated donors, and COVID-19-recovered vaccinated donors, were provided through the "Collection of Biospecimens from Persons Under Investigation for 2019-Novel Coronavirus Infection to Understand Viral Shedding and Immune Response Study" UCSD IRB\# 200236 as reported earlier (35). The protocol was approved by the UCSD Human Research Protection Program. Convalescent donor samples were collected based on COVID-19 diagnosis regardless of gender, race, ethnicity, disease severity, or other medical conditions. All human donors were assessed for medical decision-making capacity using a standardized, approved assessment, and voluntarily gave informed consent prior to being enrolled in the study.

\section{Plasmid construction}

To generate soluble $S$ ectodomain proteins from SARS-CoV-1 (residues 1-1190; GenBank: AAP13567) ,SARS-CoV-2 (residues 1-1208; GenBank: MN908947), HCoVHKU1 (residue 1-1295; GenBank: YP_173238.1), HCoV-OC43 (residues 1-1300; GenBank: AAX84792.1), MERS-CoV (residues 1-1291; GenBank: APB87319.1), HCoV229E (residues 1-1110; GenBank: NP_073551.1) and HCoV-NL63 (residues 1-1291; GenBank: YP_003767.1), we synthesized the DNA fragments from GeneArt (Life Technologies) and cloned them into the phCMV3 vector (Genlantis cat.\# P003300). In order to produce the stable trimeric prefusion spike proteins, double proline substitutions (2P) were introduced into the S2 subunit: K968/V969 in SARS-CoV-1, K986/V987 in SARS-CoV-2, V1060/L1061 in MERS-CoV, A1071/L1072 in HCoV-HKU1, A1078/L1079 in HCoV-OC43, S1052/l1053 in HCoV-NL63 and T871//872 in HCoV-229E were replaced by proline. The furin cleavage sites (in SARS-CoV-2 residues 682-685, in SARS-CoV-1 residues 664-667, in HCoV-HKU1 residues 756-760, in HCoV-OC43 residues 762-766, in MERS-CoV residues 748-751, in HCoV-229E residues 564-567 and in HCoV-NL63 residues 745-748) were replaced by a "GSAS" linker; the trimerization T4 fibritin motif was incorporated at the $\mathrm{C}$-terminus of the $\mathrm{S}$ proteins. To purify and biotinylate the spike proteins, the HRV-3C protease cleavage site, 6x HisTag, and AviTag spaced by GSlinkers were added to the C-terminus after the trimerization motif. To generate pseudoviruses of MERS-CoV and sarbecoviruses, the DNA fragments encoding the spikes of MERS-CoV and sarbecoviruses without the ER retrieval signal were codonoptimized and synthesized at GeneArt (Life Technologies). The spike encoding genes of Pang17 (residues 1-1249, GenBank: QIA48632.1), WIV1 (residues 1-1238, GenBank: KF367457) and SHC014 (residue 1-1238, GenBank: AGZ48806.1) were constructed into the phCMV3 vector (Genlantis cat.\# P003300) using the Gibson assembly (New England Biolabs, cat.\# E2621L) according to the manufacturer's instructions.

\section{Cell lines}

FreeStyle293-F cells (Thermo Fisher Scientific cat.\# R79007) were grown in FreeStyl 293 Expression Medium (Gibco cat.\# 12338018), and Expi293F cells (Gibco cat.\# A14527) were maintained in Expi293 Expression Medium (Gibco cat.\# A1435101). Suspension cells were incubated in the shaker at $150 \mathrm{rpm}, 37^{\circ} \mathrm{C}, 8 \% \mathrm{CO}_{2}$. Adherent HEK293T cells 
and HeLa-ACE2 cells were grown in Dulbecco's Modified Eagle Medium (DMEM) with $10 \%$ heat-inactivated FBS, 4mM L-Glutamine and 1\% P/S, maintaining in the incubator at $37^{\circ} \mathrm{C}, 5 \% \mathrm{CO}_{2}$. The stable hACE2 or hDPP4-expressing HeLa cell line was generated using an ACE2 lentivirus protocol previously described (7). Briefly, the pBOB-hACE2 or hDPP4 plasmid and lentiviral packaging plasmids (pMDL, pREV, and pVSV-G (Addgene \#12251, \#12253, \#8454)) were co-transfected into HeLa cells using Lipofectamine 2000 reagent (ThermoFisher Scientific cat.\# 11668019).

\section{Expression and purification of HCoV S-proteins}

To express the soluble human coronavirus (HCoV) S ectodomain proteins with His-tag or with both His- and Avi-tag at the C-terminus, $350 \mu \mathrm{g}$ plasmids in $15 \mathrm{ml}$ Opti-MEM $^{\mathrm{TM}}$ (Thermo Fisher Scientific cat.\# 31985070) were filtered and mixed with $1.8 \mathrm{ml} 40 \mathrm{~K} \mathrm{PEI}$ $(1 \mathrm{mg} / \mathrm{ml})$ in $15 \mathrm{ml}$ Opti-MEM ${ }^{\mathrm{TM}}$, then incubated at room temperature for $30 \mathrm{~min}$ and transferred into $1 \mathrm{~L}$ FreeStyle293-F cells at the density of 1 million cells $/ \mathrm{ml}$. Four days after transfection, the cell cultures were centrifuged at 2500xg for 15 min and filtered through $0.22 \mu \mathrm{m}$ membrane. The His-tagged proteins were purified with the HisPur Ni-NTA Resin (Thermo Fisher Scientific cat.\# 88221). After washing by wash buffer (25 mM Imidazole, $\mathrm{pH}$ 7.4) for at least 3 bed volumes, the protein was eluted with $25 \mathrm{ml}$ elution buffer (250 $\mathrm{mM}$ Imidazole, $\mathrm{pH} 7.4$ ) at slow gravity speed ( 4 sec/drop), then was buffer exchanged into PBS and concentrated using 100K Amicon tubes (Millipore cat.\# UFC910024). After being further purified by size-exclusion chromatography by Superdex 200 Increase 10/300 GL column (GE Healthcare cat.\# GE28-9909-44), the protein was pooled and concentrated again for further use.

\section{Flow cytometry B cell profiling and monoclonal antibody isolation}

Flow cytometry of PBMC samples from infected-vaccinated human donors were conducted following methods described previously (7). 10ml RPMI1640 medium (Thermo Fisher Scientific, cat.\# 11875085) with $50 \%$ FBS was pre-warmed to $37^{\circ} \mathrm{C}$ and used to thaw the frozen PBMC samples, followed by centrifugation at $400 \mathrm{xg}$ for $5 \mathrm{~min}$. After discarding supernatant, the cells were resuspended in a $5 \mathrm{ml} \mathrm{FACS} \mathrm{buffer} \mathrm{(PBS,} \%$ FBS, $2 \mathrm{mM}$ EDTA). Fluorescently labeled antibodies specific for cell surface markers were prepared as 1:100 dilution as a master mix in FACS buffer, to stain the PBMC samples for CD3 (APC-Cy7, BD Pharmingen cat.\# 557757), CD4 (APC-Cy7, Biolegend cat.\# 317418), CD8 (APC-Cy7, BD Pharmingen cat.\# 557760), CD14 (APC-H7, BD Pharmingen cat.\# 561384), CD19 (PerCP-Cy5.5, Fisher Scientific cat.\# NC9963455), CD20 (PerCP-Cy5.5, Biolegend, cat.\# 302326), IgG (BV605, BD Pharmingen cat.\# 563246) and IgM (PE, Biolegend, cat.\# 314508). Meanwhile, SARS-CoV-2 S protein with Avi-tag was conjugated to streptavidin-BV421 (BD Pharmingen cat.\# 563259) and streptavidin-AF488 (Invitrogen cat.\# S11223), respectively, and the MERS-CoV S protein with Avi-tag was conjugated to streptavidin-AF647 (Invitrogen cat.\# S21374). After incubating the cells with Ab mixture for cell surface markers for 15 min in dark, S proteinprobes were added to the samples and incubated on ice in the dark for $30 \mathrm{~min}$. FVS510 Live/Dead stain (Thermo Fisher Scientific cat.\# L34966) in FACS buffer (1:300) was then added to the samples and incubated on ice in the dark for $15 \mathrm{~min}$. After washing with

562 FACS buffer, the stained cells were resuspended in $500 \mu$ of FACS buffer per 10-20 563 million cells, filtered through the 70- $\mu$ m mesh cap into FACS tubes (Fisher Scientific cat.\# 
564

565

566

567

568

569

570

571

572

573

574

575

576

577

578

579

580

581

582

583

584

585

586

587

588

589

590

591

592

593

594

595

596

597

598

599

600

601

602

603

604

605

606

607

608

609

08-771-23) and sorted for S protein-specific memory B cells using BD FACSMelody sorter. In brief, after gating of lymphocytes (SSC-A vs. FSC-A) and singlets (SSC-W vs SSC-H and FSC-H vs. FSC-W), live cells were identified by the negative FVS510 live/dead staining phenotype. The CD3- CD4- CD8 CD14- CD19+ ${ }^{-}$D20 ${ }^{+}$cells were gated as $B$ cells. By selecting the $\lg G^{+} \operatorname{lgM}$ - population, the cells were sequentially gated for SARS-CoV-2-S-BV421+ SARS-CoV-2-S-AF488+ MERS-CoV-S-AF647+ reactivity. Triple positive memory $B$ cells was sorted as single cells into 96 -well plates on a cooling platform. Superscript IV Reverse Transcriptase (Invitrogen cat.\# 18090010), 10mM dNTPs (Invitrogen cat.\# 18427088), random hexamers (Gene Link cat.\# 26-4000-03), Ig gene-specific primers, 0.1M DTT, RNAseOUT (Invitrogen cat.\# 10777019), and 10\% lgepal (Sigma-Aldrich cat.\# 18896) were used in the reverse transcription PCR reaction to generate cDNA from the sorted cells right after sorting. Hot Start DNA Polymerases ((QIAGEN cat.\# 203643) and specific primer sets described previously $(78,79)$ were used to perform two rounds of nested PCR reactions to amplify IgG heavy and light chain variable regions using cDNAs as template. After being purified with SPRI beads according to manufacturer's instructions (Beckman Coulter cat.\# B23318), PCR products were constructed into expression vectors encoding human IgG1 or lg kappa/lambda constant domains, respectively, by Gibson assembly (New England Biolabs cat.\# E2621L), then transformed into competent E.coli cells. Single colonies were picked for sequencing and analysis on IMGT V-Quest online tool (http://www.imgt.org) and downstream plasmid production.

\section{Expression and purification of monoclonal antibodies}

Plasmids of the paired heavy and light chains generated after sorting were co-transfected into Expi293F cells to produce monoclonal antibodies. Briefly, $12 \mu \mathrm{g}$ heavy chain plasmid and $12 \mu \mathrm{g}$ of light chain plasmid were added into $3 \mathrm{ml}$ of Opti-MEM ${ }^{\mathrm{TM}}$ (Thermo Fisher Scientific cat.\# 31985070), after inverting, 24 $\mu$ l of FectoPRO (Polyplus cat.\# \#116-001) reagent was added into the mixture and inverted. Incubation at room temperature for $10 \mathrm{~min}$ was done before adding the mixture into $30 \mathrm{ml}$ of Expi293F cells at 2.8 million cells $/ \mathrm{ml}$ and incubating in the shaker. 24 hours after transfection, $300 \mu \mathrm{l}$ of $300 \mathrm{mM}$ sodium valproic acid solution and $275 \mu \mathrm{l}$ of $45 \%$ Glucose solution was used to feed each cell culture. Four days post transfection, supernatants of cell cultures were collected by centrifugation at $2500 \mathrm{xg}$ for $15 \mathrm{~min}$ and filtering through $0.22 \mu \mathrm{m}$ membrane. Protein $\mathrm{A}$ Sepharose (GE Healthcare cat.\# 45002982) and Protein G Sepharose (GE Healthcare cat.\# 45000118) were mixed at 1:1 ratio before adding into the supernatant and rotating overnight at $4^{\circ} \mathrm{C}$. The solution was then loaded into Econo-Pac columns (BioRad cat.\# 7321010), washed with 1 column volume of PBS, and antibodies were eluted with $10 \mathrm{ml}$ of $0.2 \mathrm{M}$ citric acid ( $\mathrm{pH} 2.67$ ). The elution was collected into a tube containing $1 \mathrm{ml}$ of $2 \mathrm{M}$ Tris Base solution. 30K Amicon centrifugal filters (Millipore cat.\# UFC903024) were used for buffer exchange into PBS and further concentrating into smaller volumes.

\section{ELISA using peptides or recombinant proteins}

$\mathrm{N}$-terminal biotinylated peptides corresponding to stem helix of SARS-CoV-1/2, MERSCoV, HCoV-HKU1, HCoV-OC43, HCoV-229E and HCoV-NL63 were synthesized at A\&A Labs (Synthetic Biomolecules) (44). For peptide ELISA, streptavidin (Jackson Immuno Research Labs cat.\# 016-000-084) was coated at $2 \mu \mathrm{g} / \mathrm{ml}$ in PBS onto 96-well half-area 
high binding plates (Corning, 3690) overnight at $4^{\circ} \mathrm{C}$. For recombinant protein ELISA, mouse anti-His antibody (Invitrogen cat. \# MA1-21315-1MG) was used at the same concentration to coat the plates. After washing by $0.05 \%$ PBST 3 times, 3\% BSA was used to block the plates for $2 \mathrm{~h}$ at $37^{\circ} \mathrm{C}$. Then $1 \mu \mathrm{g} / \mathrm{ml}$ of $\mathrm{N}$-terminal biotinylated peptide or $2 \mu \mathrm{g} / \mathrm{ml}$ of His-tagged recombinant spike proteins were applied to plates and incubated for $1 \mathrm{~h}$ at RT. After washing by $0.05 \%$ PBST 3 times, serially diluted serum samples or antibodies were added into plates and incubated for $1 \mathrm{~h}$ at RT. After another washing, alkaline phosphatase-conjugated goat anti-human IgG Fc secondary antibody (Jackson ImmunoResearch cat.\# 109-055-008) was added in 1:1000 dilution and incubated for $1 \mathrm{~h}$ at RT. After the final wash, phosphatase substrate (Sigma-Aldrich cat.\# S0942-200TAB) dissolved in staining buffer was added into each well. Absorption was measured at 405 $\mathrm{nm}$. Fifty percent maximal response concentrations (EC50) were calculated using the Asymmetrical dose-response model of Richard's version in GraphPad Prism 7 (GraphPad Software). To identify critical residues for antibody binding, single alanine mutations were introduced onto the 25-mer stem helix peptide that comprises the linear epitope. These peptides were synthesized at A\&A Labs (Synthetic Biomolecules). ELISA as described above was used to test antibody reactivity against peptides with single alanine substitutions.

\section{Pseudovirus production}

HIV-based lentivirus backbone plasmid pCMV-dR8.2 dvpr (Addgene \#8455), pBOBLuciferase (Addgene \#170674) were co-transfected into HEK293T cells along with fulllength or variously truncated SARS-CoV1, WIV1, SHC014, Pang17, SARS-COV2, SARS-CoV-2 variants of concern [(B.1.1.7(alpha), B.1.351 (beta), P.1 (gamma), B.1.617.2 (delta) and B.1.1.529 (Omicron)] and MERS-CoV spike using Lipofectamine 2000 (ThermoFisher Scientific cat.\# 11668019) to produce single-round infectioncompetent pseudoviruses (80). The medium was changed 12-16 hours post transfection. Pseudovirus-containing supernatants were collected 48 hours post transfection and the viral titers were measured by luciferase activity in relative light units (RLU) (Bright-Glo Luciferase Assay System, Promega cat.\# E2620). The supernatants were aliquoted and stored at $-80^{\circ} \mathrm{C}$ until further use.

\section{Neutralization assay}

Pseudotyped viral neutralization assay was performed as previously reported (7). In brief, neutralization assays were performed by adding $25 \mu \mathrm{l}$ of pseudovirus into $25 \mu \mathrm{l}$ serial dilutions of purified antibodies or plasma from human donors, the mixture was then dispensed into a 96 -well plate incubated for one hour at $37^{\circ} \mathrm{C}$, then $10,000 \mathrm{HeLa}$-hACE2 or hDPP4 cells/ well (in $50 \mu \mathrm{l}$ of media containing $20 \mu \mathrm{g} / \mathrm{ml}$ Dextran) were directly added to the mixture. After incubation at $37^{\circ} \mathrm{C}$ for $42-48 \mathrm{~h}$, luciferase activity was measured. Neutralizing activity was measured by reduction in luciferase activity compared to the virus controls. Fifty percent maximal inhibitory concentrations $\left(\mathrm{IC}_{50}\right)$, the concentrations required to inhibit infection by $50 \%$ compared to the controls, were calculated using the dose-response-inhibition model with 5-parameter Hill slope equation in GraphPad Prism 7 (GraphPad Software).

\section{Neutralization Assay of Replication Competent CoVs}


656

657

658

659

660

661

662

663

664

665

666

667

668

669

670

671

672

673

674

675

676

677

678

679

680

681

682

683

684

685

686

687

688

689

690

691

692

693

694

695

696

697

698

699

700

701

702

Vero E6 cells (ATCC-C1008) were seeded at $2 \times 10^{4}$ cells/well in a black-well, black-wall, tissue culture treated, 96-well plate (Corning Cat. \#3916) $24 \mathrm{~h}$ before the assay. Abs were diluted in MEM supplemented with 5\%FBS and 1\%Pen/Strep media to obtain an 8-point, 3-fold dilution curve with starting concentration at $20 \mu \mathrm{g} / \mathrm{ml}$. Eight hundred Pfu of SARS2-nLuc and MERS-nLuc replication competent viruses were mixed with $A$ bs at a $1: 1$ ratio and incubated at $37^{\circ} \mathrm{C}$ for $1 \mathrm{~h}$. One-hundred microliters of virus and $\mathrm{Ab}$ mix was added to each well and incubated at $37^{\circ} \mathrm{C}+5 \%$ $\mathrm{CO}_{2}$ for 20 to $22 \mathrm{~h}$. Luciferase activities were measured by the Nano-Glo Luciferase Assay System (Promega Cat. \#N1130) following the manufacturer's protocol using a GloMax luminometer (Promega). Percent inhibition and $\mathrm{IC}_{50}$ were calculated as pseudovirus neutralization assay described above. All experiments were performed as duplicate and independent repeated for three times. All the live virus experiments were performed under biosafety level 3 (BSL-3) conditions at negative pressure, by operators in Tyvek suits wearing personal powered-air purifying respirators.

\section{HEp2 epithelial cell polyreactive assay}

According to manufacturer's instructions, HEp2 slides (Hemagen cat.\# 902360) were used to determine the reactivity of monoclonal antibodies to human epithelial type 2 (HEp2) by indirect immunofluorescence. Briefly, monoclonal antibody was diluted into $50 \mu \mathrm{g} / \mathrm{ml}$ by PBS and then added onto immobilized HEp2 slides and incubated for 30 min at RT. After washing by PBS for 3 times, one drop of FITC-conjugated goat anti-human $\operatorname{lgG}$ was added onto each well and incubated in the dark for 30 min at RT. After washing, the coverslip was added to HEp2 slide with glycerol and the images were photographed on a Nikon fluorescence microscope for FITC detection.

\section{Polyspecificity reagent (PSR) ELISA}

Solubilized $\mathrm{CHO}$ cell membrane protein (SMP), human insulin (Sigma-Aldrich cat.\# I2643), single strand DNA (Sigma-Aldrich cat.\# D8899) were coated onto 96-well halfarea high-binding plates (Corning cat.\# 3690) at $5 \mu \mathrm{g} / \mathrm{ml}$ in PBS overnight at $4^{\circ} \mathrm{C}$. After washing with PBST, plates were blocked with $3 \% \mathrm{BSA}$ for $2 \mathrm{~h}$ at $37^{\circ} \mathrm{C}$. Antibody samples were diluted at $50 \mu \mathrm{g} / \mathrm{ml}$ in $1 \%$ BSA with 5 -fold serial dilution and then added in plates to incubate for $1 \mathrm{~h}$ at room temperature (7). The assay was performed as described in section "ELISA using peptides or recombinant proteins".

\section{CELISA binding}

Flow cytometry-based Cell-ELISA (CELISA) binding of mAbs with HCoV spikes was performed as described previously $(43,81)$. A total of $4 \times 10^{6} \mathrm{HEK} 293 \mathrm{~T}$ cells were seeded into $10 \mathrm{~cm}$ round cell culture dishes and incubated at $37^{\circ} \mathrm{C}$. After $24 \mathrm{~h}, \mathrm{HEK} 293 \mathrm{~T}$ cells were transfected with plasmids encoding full-length HCoV spikes and were incubated for 36$48 \mathrm{~h}$ at $37^{\circ} \mathrm{C}$. The cells were harvested and distributed into 96 -well round-bottom tissue culture plates for individual staining reactions. For each staining reaction, cells were

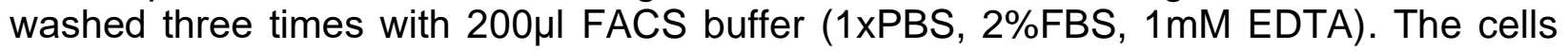
were stained for $1 \mathrm{~h}$ on ice in $50 \mu \mathrm{l}$ staining buffer with $10 \mu \mathrm{g} / \mathrm{ml}$ of primary antibody. After

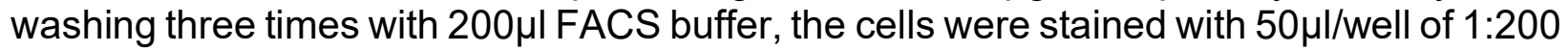
diluted R-phycoerythrin (PE)-conjugated mouse anti-human IgG Fc antibody (SouthernBiotech cat.\# 9040-09) and 1:1000 dilution of Zombie-NIR viability dye (BioLegend cat.\# 423105) on ice in dark for 45min. Following three washes with FACS buffer, the cells were resuspended and analyzed by flow cytometry (BD Lyrics cytometer), 
703

704

705

706

707

708

709

710

711

712

713

714

715

716

717

718

719

720

721

722

723

724

725

726

727

728

729

730

731

732

733

734

735

736

737

738

739

740

741

742

743

744

745

746

747

748

and the binding data were generated by calculating the Mean Fluorescence Intensity using FlowJo 10 software. Mock-transfected 293T cells were used as a negative control.

\section{BioLayer Interferometry binding (BLI)}

Octet K2 system (ForteBio) was used to determine the monoclonal antibody binding with S-proteins or selected peptides. IgG was first captured for 60 s by anti-human IgG Fc capture (AHC) biosensors (ForteBio cat.\# 18-5063), then baseline was provided in Octet buffer (PBS with $0.1 \%$ Tween) for another 60s. After that, the sensors were transferred into wells containing diluted HCoV S-proteins for 120 s for association, and into Octet buffer for disassociation for 240s. Selected peptides that were N-terminal biotinylated were diluted in Octet buffer and first captured for 60 s by the hydrated streptavidin biosensors (ForteBio cat.\# 18-5020), then unbound peptides were removed by transferring into Octet buffer for 60s to provide the baseline. Then the sensors were immersed into monoclonal antibodies in Octet buffer for 120 s for association, followed by transferring into Octet buffer for 240 s for dissociation. The data generated were analyzed using the ForteBio Data Analysis software for correction, and the kinetic curves were fit to $1: 1$ binding mode. Note that the $\mathrm{lgG}$ : spike protomer binding can be a mixed population of $2: 1$ and $1: 1$, such that the term 'apparent affinity' dissociation constants $\left(\mathrm{K}_{D}^{\mathrm{App}}\right)$ are shown to reflect the binding affinity between IgGs and spike trimers tested.

\section{Antibody immunogenetics analysis}

Heavy and light chain sequences of mature antibodies were processed using DiversityAnalyzer tool (82). For each $\mathrm{CDRH} 3$ translated in the amino acid alphabet, all its $k$-mers were extracted, where $k=2,3,4$. K-mers appearing in at least $20 \%$ of HCDR3s were reported as motifs. In total 10 motifs were reported for CDRH3s: AR, ARG, AS, DY, FD, FDY, GS, GV, RG, SS. Each heavy chain sequence was labeled by whether its $\mathrm{CDRH} 3$ contains a given motif. The same procedure was applied to CDRL3s and reported 16 motifs: DS, DSS, FT, GS, PP, QQ, QQY, QY, QYG, SP, SPP, SS, SSP, SSPP, WD, YG. For each CDRH3 motif, the linear regression model was applied to estimate the impact of the motif presence (denoted as "yes" or "no") and the type of antibody (denoted as "iGL" or "mature") on the responses of 32 mature antibodies to the stem helix peptides of SARS-CoV-2 and MERS-CoV viruses. The same method was applied to estimate the impact of the presence of LCDR3 motifs. Heavy and light chain sequences of the same antibody were concatenated into a single sequence and collected across all 32 antibodies. The phylogenetic tree derived from the concatenated sequences was constructed using ClusterW2 tool (83) and visualized using the Iroki tool (84).

\section{In vivo virus challenge in mouse model}

All mouse experiments were performed at the University of North Carolina, NIH/PHS Animal Welfare Assurance Number: D16-00256 (A3410-01), under approved IACUC protocols. The animal manipulation and virus work was performed in a Class $2 A$ biological safety cabinet in a BSL3 approved facility and workers wore PAPRs, tyvek suites and were double gloved. 12-month-old female Balb/c mice (strain 047) were purchased from Envigo for Sarbecovirus challenge experiments (65, 85). C57Bl/6 288/330+/+ mice, which encode two human codons in the mouse dipeptidyl peptidase gene, were used for MERSCoV mouse adapted challenge experiments (66). Mice were housed in individually 
ventilated Seal-Safe cages, provided food and water ad libitum and allowed to acclimate at least seven days before experimental use. Twelve hours prior to infection, $300 \mu \mathrm{g}$ antibody was injected into mice intraperitoneally. Immediately prior to infection, mice were anesthetized by injection of ketamine and xylazine intraperitoneally and weighed. Virus (SARS-CoV MA15, SARS-CoV2 MA10 and mouse adapted MERS-CoV-M35c4) was diluted in 50 $\mu$ l sterile PBS and administered intranasally (65-67, 85). Mice were weighed daily and observed for signs of disease. The mice were euthanized via isoflurane overdose at the designated timepoint, followed by assessment of gross lung pathology and collection of the inferior lobe for virus titration. Respiratory function was measured at day2 post infection via Buxco whole body plethysmography, as previously described (86).

\section{Virus titration}

761 SARS-CoV-2-MA10, SARS-CoV-1-MA15 and MERS-CoV-M35c4 were grown and titered using VeroE6 cells as previously described (87). Briefly, lung tissue was homogenized in $1 \mathrm{ml}$ sterile PBS via Magnalyser (Roche), centrifuged to pellet debris, plated in 10-fold serial dilutions on VeroE6 cells on a 6 -well plate and covered with a 1:1 mixture of $1.6 \%$ agarose and media. At two (SARS-CoV-1) or three (SARS-CoV-2) days post plating, cells were stained with neutral red and plaques counted.

\section{Statistical Analysis}

Statistical analysis was performed using Graph Pad Prism 8, Graph Pad Software, San Diego, California, USA. ID 50 or $\mathrm{IC}_{50}$ titers were compared using the non-parametric unpaired Mann-Whitney- $U$ test. The correlation between two groups was determined by Spearman rank test. Groups of data were compared using the Kruskal-Wallis nonparametric test. Dunnett's multiple comparisons test were also performed between experimental groups. Data were considered statistically significant at $p<0.05$. 


\section{References}

1. L. R. Baden et al., Efficacy and Safety of the mRNA-1273 SARS-CoV-2 Vaccine. The New England journal of medicine 384, 403-416 (2020).

2. F. P. Polack et al., Safety and Efficacy of the BNT162b2 mRNA Covid-19 Vaccine. The New England journal of medicine 383, 2603-2615 (2020).

3. Z. Wang et al., mRNA vaccine-elicited antibodies to SARS-CoV-2 and circulating variants. Nature 592, 616-622 (2021).

4. P. B. Gilbert et al., Title: Immune Correlates Analysis of the mRNA-1273 COVID-19 Vaccine Efficacy Trial. Science 375, 43-50 (2021).

5. A. J. Greaney et al., Antibodies elicited by mRNA-1273 vaccination bind more broadly to the receptor binding domain than do those from SARS-CoV-2 infection. Science translational medicine 13, eabi9915 (2021).

6. C. O. Barnes et al., Structures of Human Antibodies Bound to SARS-CoV-2 Spike Reveal Common Epitopes and Recurrent Features of Antibodies. Cell 182, 828-842 e816 (2020).

7. T. F. Rogers et al., Isolation of potent SARS-CoV-2 neutralizing antibodies and protection from disease in a small animal model. Science 369, 956-963 (2020).

8. M. Yuan et al., Structural basis of a shared antibody response to SARS-CoV-2. Science 369, 1119-1123 (2020).

9. D. F. Robbiani et al., Convergent antibody responses to SARS-CoV-2 in convalescent individuals. Nature 584, 437-442 (2020).

10. P. J. M. Brouwer et al., Potent neutralizing antibodies from COVID-19 patients define multiple targets of vulnerability. Science 369, 643-650 (2020).

11. S. J. Zost et al., Potently neutralizing and protective human antibodies against SARSCoV-2. Nature 584, 443-449 (2020).

12. C. O. Barnes et al., SARS-CoV-2 neutralizing antibody structures inform therapeutic strategies. Nature 588, 682-687 (2020).

13. Z. Wang et al., Naturally enhanced neutralizing breadth against SARS-CoV-2 one year after infection. Nature 595, 426-431 (2021).

14. M. Yuan et al., Structural and functional ramifications of antigenic drift in recent SARSCoV-2 variants. Science 373, 818-823 (2021).

15. P. Wang et al., Antibody Resistance of SARS-CoV-2 Variants B.1.351 and B.1.1.7. Nature 593, 130-135 (2021).

16. J. R. Mascola, B. S. Graham, A. S. Fauci, SARS-CoV-2 Viral Variants-Tackling a Moving Target. JAMA 325, 1261-1262 (2021).

17. W. T. Harvey et al., SARS-CoV-2 variants, spike mutations and immune escape. Nat Rev Microbiol 19, 409-424 (2021).

18. E. Cameroni et al., Broadly neutralizing antibodies overcome SARS-CoV-2 Omicron antigenic shift. Nature, (2021).

19. L. Liu et al., Striking Antibody Evasion Manifested by the Omicron Variant of SARS-CoV2. Nature, (2021).

20. S. Cele et al., Omicron extensively but incompletely escapes Pfizer BNT162b2 neutralization. Nature, (2021).

21. D. Planas et al., Considerable escape of SARS-CoV-2 Omicron to antibody neutralization. Nature, (2021).

22. J. M. Carreno et al., Activity of convalescent and vaccine serum against SARS-CoV-2 Omicron. Nature, (2021).

23. W. F. Garcia-Beltran et al., mRNA-based COVID-19 vaccine boosters induce neutralizing immunity against SARS-CoV-2 Omicron variant. Cell, (2022). 
827

828

829

830

831

832

833

834

835

836

837

838

839

840

841

842

843

844

845

846

847

848

849

850

851

852

853

854

855

856

857

858

859

860

861

862

863

864

865

866

867

868

869

870

871

872

873

874

875

876

877

24. C. K. Wibmer et al., SARS-CoV-2 501Y.V2 escapes neutralization by South African COVID-19 donor plasma. Nature medicine 27, 622-625 (2021).

25. Y. Cao et al., Omicron escapes the majority of existing SARS-CoV-2 neutralizing antibodies. Nature, (2021).

26. M. Letko, A. Marzi, V. Munster, Functional assessment of cell entry and receptor usage for SARS-CoV-2 and other lineage B betacoronaviruses. Nat Microbio/ 5, 562-569 (2020).

27. V. D. Menachery et al., SARS-like WIV1-CoV poised for human emergence. Proceedings of the National Academy of Sciences of the United States of America 113, 3048-3053 (2016).

28. A. M. Zaki, S. van Boheemen, T. M. Bestebroer, A. D. Osterhaus, R. A. Fouchier, Isolation of a novel coronavirus from a man with pneumonia in Saudi Arabia. The New England journal of medicine 367, 1814-1820 (2012).

29. D. R. Burton, E. J. Topol, Variant-proof vaccines - invest now for the next pandemic. Nature 590, 386-388 (2021).

30. W. C. Koff, S. F. Berkley, A universal coronavirus vaccine. Science 371, 759 (2021).

31. D. M. Morens, J. K. Taubenberger, A. S. Fauci, Universal Coronavirus Vaccines - An Urgent Need. The New England journal of medicine, (2021).

32. T. N. Starr et al., SARS-CoV-2 RBD antibodies that maximize breadth and resistance to escape. Nature 597, 97-102 (2021).

33. M. A. Tortorici et al., Broad sarbecovirus neutralization by a human monoclonal antibody. Nature 597, 103-108 (2021).

34. D. Pinto et al., Cross-neutralization of SARS-CoV-2 by a human monoclonal SARS-CoV antibody. Nature 583, 290-295 (2020).

35. W.-t. He et al., Targeted isolation of panels of diverse human broadly neutralizing antibodies against SARS-like viruses. bioRxiv, 2021.2009.2008.459480 (2021).

36. D. R. Martinez et al., A broadly cross-reactive antibody neutralizes and protects against sarbecovirus challenge in mice. Science translational medicine, eabj7125 (2021).

37. M. Yuan et al., A highly conserved cryptic epitope in the receptor binding domains of SARS-CoV-2 and SARS-CoV. Science 368, 630-633 (2020).

38. C. A. Jette et al., Broad cross-reactivity across sarbecoviruses exhibited by a subset of COVID-19 donor-derived neutralizing antibodies. bioRxiv, (2021).

39. W.-t. He et al., Broadly neutralizing antibodies to SARS-related viruses can be readily induced in rhesus macaques. bioRxiv, 2021.2007.2005.451222 (2021).

40. D. Li et al., In vitro and in vivo functions of SARS-CoV-2 infection-enhancing and neutralizing antibodies. Cell 184, 4203-4219 e4232 (2021).

41. C. G. Rappazzo et al., Broad and potent activity against SARS-like viruses by an engineered human monoclonal antibody. Science 371, 823-829 (2021).

42. L. Dai, G. F. Gao, Viral targets for vaccines against COVID-19. Nature reviews. Immunology 21, 73-82 (2020).

43. G. Song et al., Cross-reactive serum and memory B-cell responses to spike protein in SARS-CoV-2 and endemic coronavirus infection. Nature communications 12, 2938 (2021).

44. P. Zhou et al., A protective broadly cross-reactive human antibody defines a conserved site of vulnerability on beta-coronavirus spikes. bioRxiv, (2021).

45. D. Pinto et al., Broad betacoronavirus neutralization by a stem helix-specific human antibody. Science 373, 1109-1116 (2021).

46. C. L. Hsieh et al., Stabilized coronavirus spike stem elicits a broadly protective antibody. Cell reports 37, 109929 (2021).

47. N. K. Hurlburt et al., Structural definition of a pan-sarbecovirus neutralizing epitope on the spike S2 subunit. bioRxiv, 2021.2008.2002.454829 (2021). 
878

879

880

881

882

883

884

885

886

887

888

889

890

891

892

893

894

895

896

897

898

899

900

901

902

903

904

905

906

907

908

909

910

911

912

913

914

915

916

917

918

919

920

921

922

923

924

925

926

927

928

48. M. M. Sauer et al., Structural basis for broad coronavirus neutralization. Nature structural \& molecular biology 28, 478-486 (2021).

49. W. Li et al., Structural basis and mode of action for two broadly neutralizing antibodies against SARS-CoV-2 emerging variants of concern. Cell reports 38, 110210 (2022).

50. C. Wang et al., A conserved immunogenic and vulnerable site on the coronavirus spike protein delineated by cross-reactive monoclonal antibodies. Nature communications $\mathbf{1 2}$, 1715 (2021).

51. M. F. Jennewein et al., Isolation and characterization of cross-neutralizing coronavirus antibodies from COVID-19+ subjects. Cell reports 36, 109353 (2021).

52. R. Andrabi, J. N. Bhiman, D. R. Burton, Strategies for a multi-stage neutralizing antibody-based HIV vaccine. Curr Opin Immunol 53, 143-151 (2018).

53. J. M. Steichen et al., A generalized HIV vaccine design strategy for priming of broadly neutralizing antibody responses. Science 366, (2019).

54. J. Jardine et al., Rational HIV immunogen design to target specific germline B cell receptors. Science 340, 711-716 (2013).

55. E. J. Erbelding et al., A Universal Influenza Vaccine: The Strategic Plan for the National Institute of Allergy and Infectious Diseases. J Infect Dis 218, 347-354 (2018).

56. D. R. Burton, L. M. Walker, Rational Vaccine Design in the Time of COVID-19. Cell host \& microbe 27, 695-698 (2020).

57. A. C. Hurt, A. K. Wheatley, Neutralizing Antibody Therapeutics for COVID-19. Viruses 13, 628 (2021).

58. L. A. Jackson et al., An mRNA Vaccine against SARS-CoV-2 - Preliminary Report. The New England journal of medicine 383, 1920-1931 (2020).

59. J. Sadoff et al., Safety and Efficacy of Single-Dose Ad26.COV2.S Vaccine against Covid-19. The New England journal of medicine 384, 2187-2201 (2021).

60. C. Soto et al., High frequency of shared clonotypes in human B cell receptor repertoires. Nature 566, 398-402 (2019).

61. B. Briney, A. Inderbitzin, C. Joyce, D. R. Burton, Commonality despite exceptional diversity in the baseline human antibody repertoire. Nature 566, 393-397 (2019).

62. M. Gidoni et al., Mosaic deletion patterns of the human antibody heavy chain gene locus shown by Bayesian haplotyping. Nature communications 10, 628 (2019).

63. R. Andrabi et al., Identification of Common Features in Prototype Broadly Neutralizing Antibodies to HIV Envelope V2 Apex to Facilitate Vaccine Design. Immunity 43, 959-973 (2015).

64. R. Andrabi et al., The Chimpanzee SIV Envelope Trimer: Structure and Deployment as an HIV Vaccine Template. Cell reports 27, 2426-2441 e2426 (2019).

65. S. R. Leist et al., A Mouse-Adapted SARS-CoV-2 Induces Acute Lung Injury and Mortality in Standard Laboratory Mice. Cell 183, 1070-1085 e1012 (2020).

66. A. S. Cockrell et al., A mouse model for MERS coronavirus-induced acute respiratory distress syndrome. Nat Microbio/ 2, 16226 (2016).

67. M. G. Douglas, J. F. Kocher, T. Scobey, R. S. Baric, A. S. Cockrell, Adaptive evolution influences the infectious dose of MERS-CoV necessary to achieve severe respiratory disease. Virology 517, 98-107 (2018).

68. M. S. Cohen, Monoclonal Antibodies to Disrupt Progression of Early Covid-19 Infection. The New England journal of medicine 384, 289-291 (2021).

69. W. A. Fischer, 2nd et al., A Phase 2a clinical trial of Molnupiravir in patients with COVID19 shows accelerated SARS-CoV-2 RNA clearance and elimination of infectious virus. Science translational medicine, eabl7430 (2021).

70. A. Schafer et al., Antibody potency, effector function, and combinations in protection and therapy for SARS-CoV-2 infection in vivo. The Journal of experimental medicine 218, (2021). 
929 71. U. Greenbaum et al., High Levels of Common Cold Coronavirus Antibodies in Convalescent Plasma Are Associated With Improved Survival in COVID-19 Patients. Frontiers in immunology 12, 675679 (2021).

72. P. Kaplonek et al., Early cross-coronavirus reactive signatures of humoral immunity against COVID-19. Sci Immunol 6, eabj2901 (2021).

73. B. F. Haynes, J. R. Mascola, The quest for an antibody-based HIV vaccine. Immunological reviews 275, 5-10 (2017).

74. P. D. Kwong, J. R. Mascola, G. J. Nabel, Broadly neutralizing antibodies and the search for an HIV-1 vaccine: the end of the beginning. Nature reviews. Immunology 13, 693-701 (2013).

75. K. O. Saunders et al., Neutralizing antibody vaccine for pandemic and pre-emergent coronaviruses. Nature 594, 553-559 (2021).

76. A. C. Walls et al., Elicitation of Potent Neutralizing Antibody Responses by Designed Protein Nanoparticle Vaccines for SARS-CoV-2. Cell 183, 1367-1382 e1317 (2020).

77. M. G. Joyce et al., A SARS-CoV-2 ferritin nanoparticle vaccine elicits protective immune responses in nonhuman primates. Science translational medicine, eabi5735 (2021).

78. T. Tiller et al., Efficient generation of monoclonal antibodies from single human B cells by single cell RT-PCR and expression vector cloning. Journal of immunological methods 329, 112-124 (2008).

79. N. A. Doria-Rose et al., New Member of the V1V2-Directed CAP256-VRC26 Lineage That Shows Increased Breadth and Exceptional Potency. Journal of virology 90, 76-91 (2016).

80. X. Zhou et al., Diverse immunoglobulin gene usage and convergent epitope targeting in neutralizing antibody responses to SARS-CoV-2. Cell reports 35, 109109 (2021).

81. P. Zhou et al., Broadly resistant HIV-1 against CD4-binding site neutralizing antibodies. PLoS pathogens 15, e1007819 (2019).

82. A. Shlemov et al., Reconstructing Antibody Repertoires from Error-Prone Immunosequencing Reads. Journal of immunology 199, 3369-3380 (2017).

83. M. A. Larkin et al., Clustal W and Clustal X version 2.0. Bioinformatics 23, 2947-2948 (2007).

84. R. M. Moore, A. O. Harrison, S. M. McAllister, S. W. Polson, K. E. Wommack, Iroki: automatic customization and visualization of phylogenetic trees. PeerJ 8, e8584 (2020).

85. M. Frieman et al., Molecular determinants of severe acute respiratory syndrome coronavirus pathogenesis and virulence in young and aged mouse models of human disease. Journal of virology 86, 884-897 (2012).

86. V. D. Menachery, L. E. Gralinski, R. S. Baric, M. T. Ferris, New Metrics for Evaluating Viral Respiratory Pathogenesis. PloS one 10, e0131451 (2015).

87. B. Yount et al., Severe acute respiratory syndrome coronavirus group-specific open reading frames encode nonessential functions for replication in cell cultures and mice. Journal of virology 79, 14909-14922 (2005). 


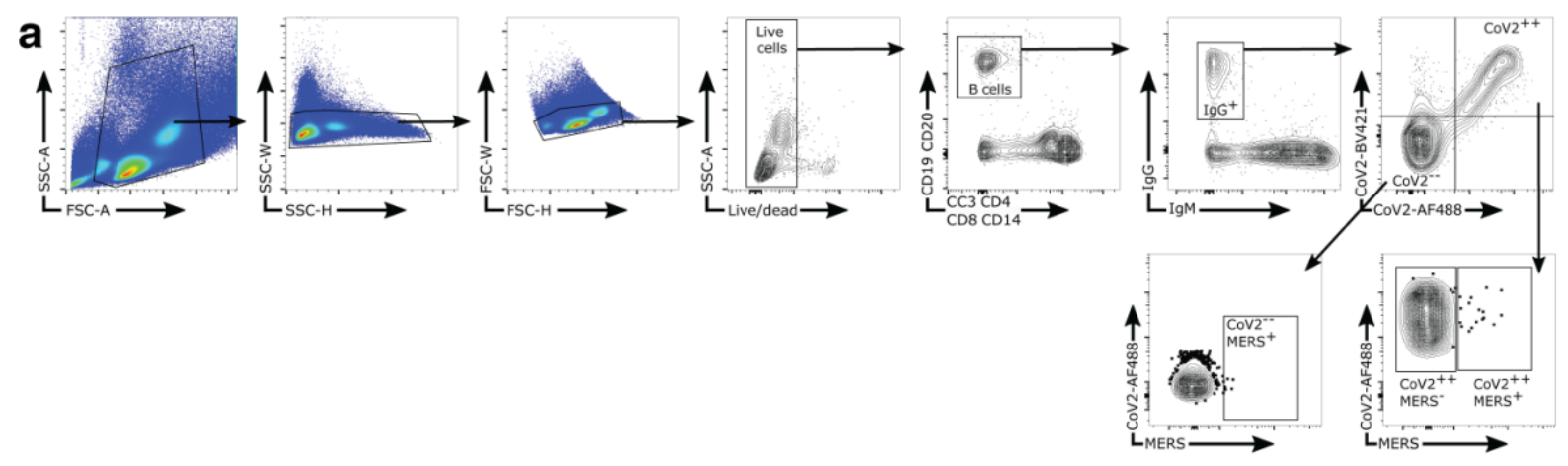

b

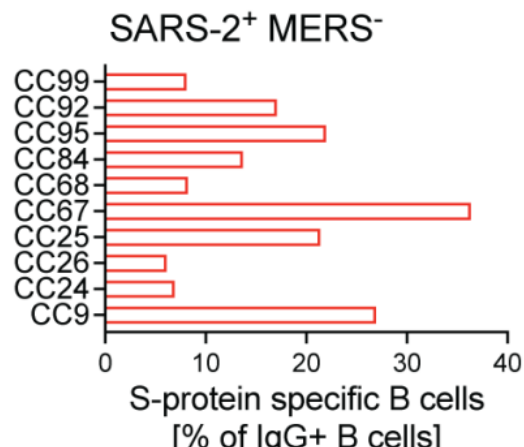

SARS-2 ${ }^{+}$MERS $^{+}$

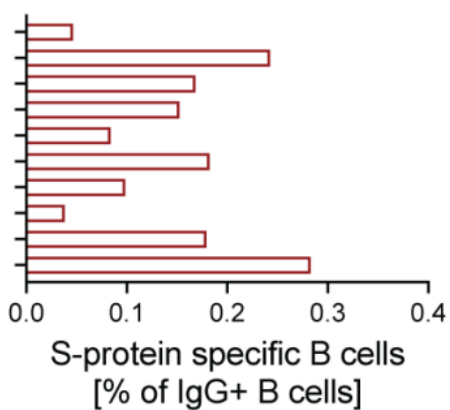

MERS ${ }^{+}$SARS-2-

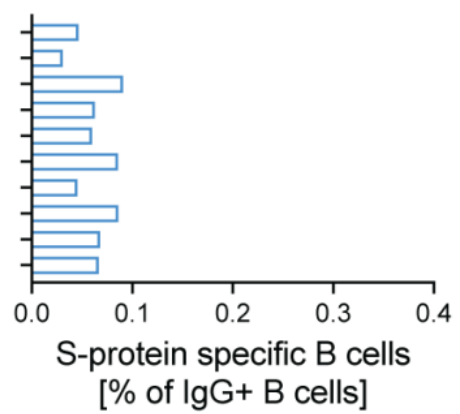

C

\begin{tabular}{|c|c|c|c|c|c|}
\hline Donor & $\begin{array}{l}\text { SARS-2/MERS spike } \\
\text { double positive B cells }\end{array}$ & $\begin{array}{c}\mathrm{H} / \mathrm{L} \text { paired antibodies } \\
\text { recovered }\end{array}$ & (\% Efficiency) & $\begin{array}{l}\text { Antibodies positive for } \\
\text { SARS-2/MERS stem helix } \\
\text { peptides binding* }\end{array}$ & $\begin{array}{l}\% \text { stem-helix } \\
\text { directed }\end{array}$ \\
\hline CC9 & 50 & 38 & 76 & 10 & 26 \\
\hline $\mathrm{CC} 24$ & 20 & 15 & 75 & 2 & 13 \\
\hline CC67 & 40 & 31 & 78 & 3 & 10 \\
\hline CC68 & 16 & 11 & 69 & 2 & 18 \\
\hline CC84 & 44 & 20 & 45 & 2 & 10 \\
\hline CC92 & 99 & 83 & 84 & 2 & 2 \\
\hline CC95 & 52 & 27 & 52 & 9 & 33 \\
\hline CC99 & 8 & 6 & 75 & 3 & 50 \\
\hline
\end{tabular}

Supplementary Figure 1. Flow cytometry B cell profiling, sorting strategy and SARS-CoV-2 and MERS-CoV S-protein specific B cells in infected-vaccinated donors. a. Gating strategy for analysis of $\mathrm{lgG}^{+} \mathrm{B}$ cell populations that bind MERS-CoV S-protein only (CD3-CD4-CD8 ${ }^{-}$CD14-CD19+CD20+lgM-lgG ${ }^{+}$CoV2-MERS-CoV ${ }^{+}$), SARS-

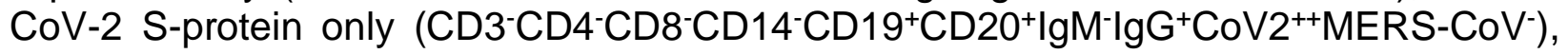
or both MERS-CoV and SARS-CoV-2 S-proteins (CD3-CD4 ${ }^{-}$CD8 ${ }^{-C D} 144^{-}{ }^{-} D 19^{+}{ }^{+}{ }^{-}{ }^{-} 0^{+}{ }^{+}$M $^{-}$ $\left.\operatorname{lgG}{ }^{+} \mathrm{CoV}_{2}{ }^{++} \mathrm{MERS}-\mathrm{CoV}^{+}\right)$. b. The frequencies of SARS-CoV-2 S-protein-specific $\operatorname{lgG}{ }^{+} \mathrm{B}$ cells (left), SARS-CoV-2 and MERS-CoV double positive S-protein-specific lgG ${ }^{+}$crossreactive $B$ cells (middle) or MERS-CoV S-protein-specific lgG ${ }^{+} B$ cells (right) in PBMCs of 10 infected vaccined-vaccinated donors. c. Summary of the number of SARS-CoV-2 and MERS-CoV double positive S-protein specific cross-reactive $B$ cells recovered from each of the donor, number and efficiency of heavy and light chain paired recovered, number of stem-helix mAb in each donor and their frequency out of the S-protein specific crossreactive IgG $B$ cells. 


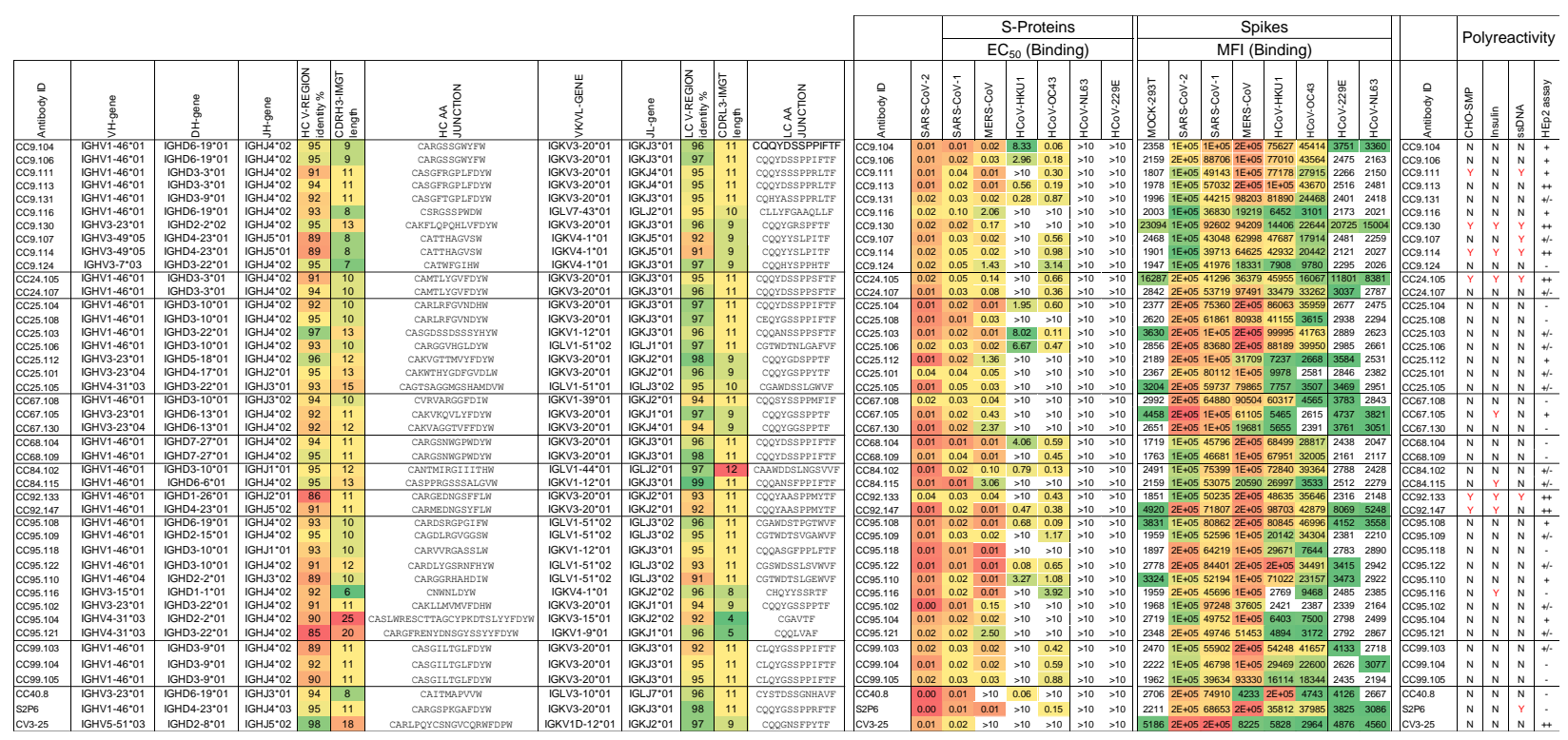
stem-helix mAbs. A total of $40 \mathrm{~S} 2$ stem-helix mAbs from 9 SARS-CoV-2 infectedvaccinated donors (CC9 $(n=10), \operatorname{CC} 24(n=2), \operatorname{CC} 25(n=7), \operatorname{CC} 67(n=3), \operatorname{CC} 68(n=$ 2), CC84 $(n=2), \operatorname{CC9}(n=2), \operatorname{CC95}(n=9)$ and CC99 $(n=3)$ were isolated by single $B$ cell sorting using SARS-CoV-2 and MERS-CoV S-proteins as baits. 32 out of $40 \mathrm{mAb}$ were encoded by unique gene families. Heavy $(\mathrm{V}, \mathrm{D}, \mathrm{J})$ and light $(\mathrm{V}, \mathrm{J})$ germline gene usage, CDR3 lengths and somatic hypermutation (SHM) levels are shown. MAbs were expressed and tested for binding to soluble (ELISA) and cell surface expressed (CellELISA) spikes derived from human $\beta$-(SARS-CoV-1 or 2, MERS-CoV, HCoV-HKU1 and $\mathrm{HCoV}-\mathrm{OC} 43)$ and $\alpha-\left(\mathrm{HCoV}-\mathrm{NL} 63\right.$ and HCoV-229E) coronaviruses and $\mathrm{EC}_{50}$ and MFI (mean fluorescent intensity) binding values are shown. S2 stem-helix bnAbs show binding to $\beta$ - but not $\alpha-\mathrm{HCoV}$ spikes. Binding to cell surface expressed spikes was relatively better compared to soluble S-proteins. Polyreactive binding analysis of S2 stem-helix bnAbs to HEp2 cells and by ELISA for binding against polyspecific reagents (PSR) including Chinese hamster ovary cells solubilized membrane protein (CHO-SMP), insulin and single-strand DNA (ssDNA). More details are included in fig. S4. S2 stem helix bnAbs, CC40.8, S2P6 and CV3-25 were used as control for binding assays. 
1005

1006

1007

1008

1009

1010

1011

1012

1013 b

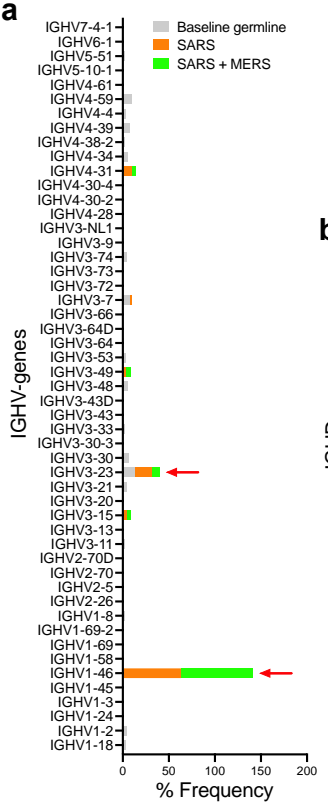

a

Supplementary Figure 3. Immunoglobulin heavy and light chain gene usage and enrichment in isolated mAbs compared to a reference human germline database. Baseline germline frequencies of heavy chain genes (IGHV, IGHD and IGHJ genes) (a., b., c) and light chain genes (IGKV, IGLV, IGKJ and IGLJ genes) (d., e) are shown in grey, and S2 stem helix sarbecovirus bnAbs (SARS: orange) and sarbecovirus + MERS-CoV bnAb (SARS + MERS: green) are shown. Arrows indicate gene enrichments compared to human baseline germline frequencies. 


\section{a}

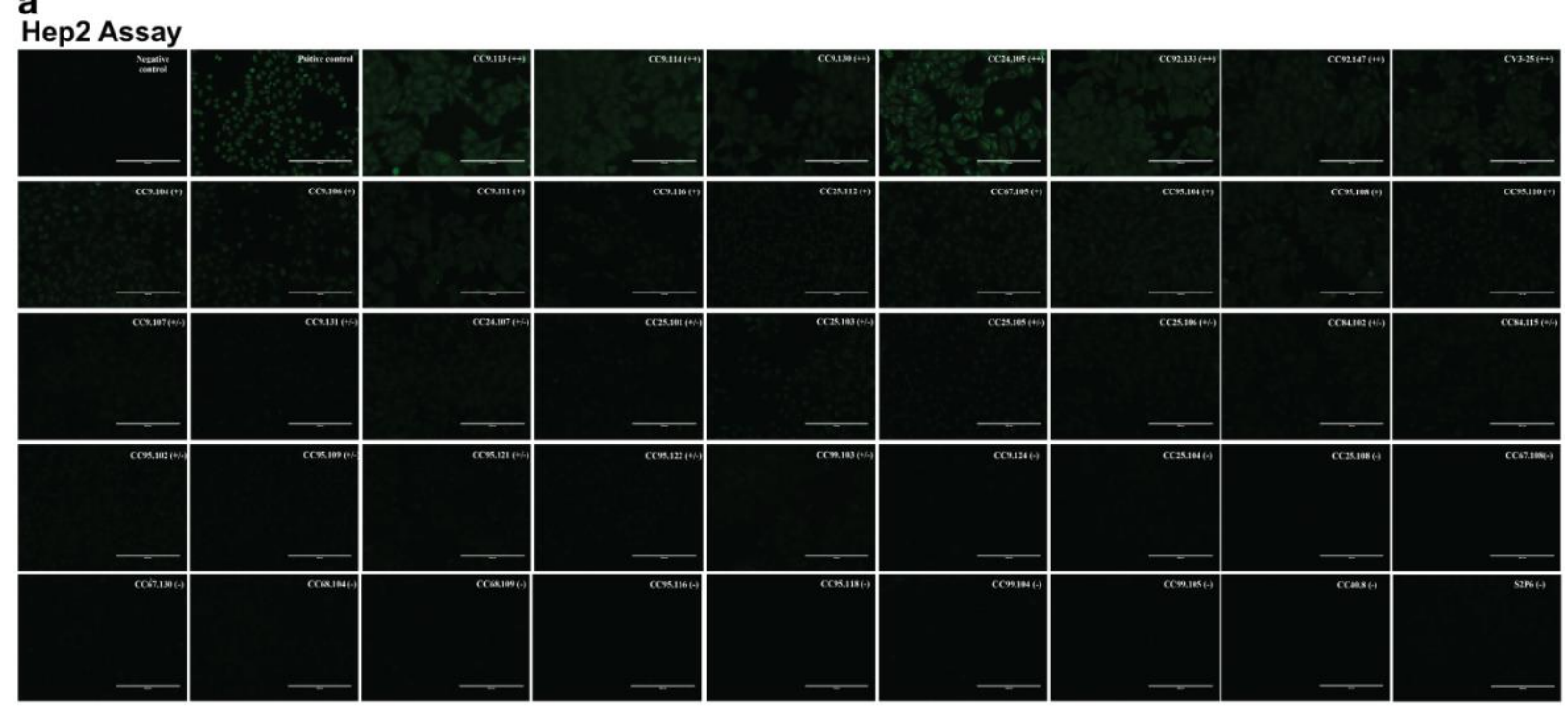

b PSR ELISA

CHO-SMP
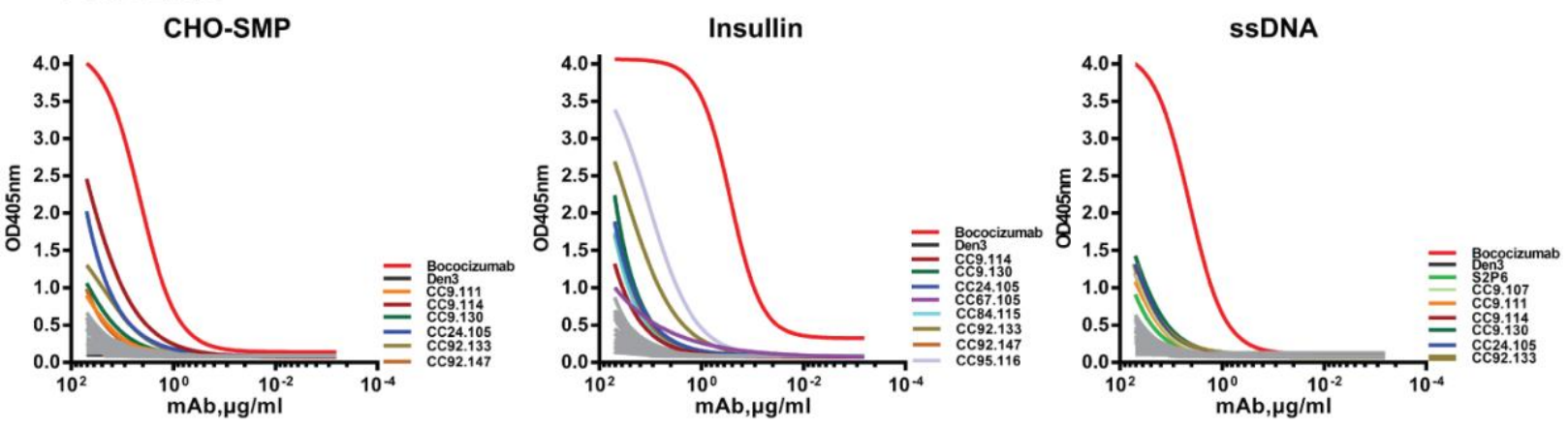

Supplementary Figure 4. Evaluation of stem-helix bnAbs for polyreactivity and autoreactivity. a-b. Antibodies were tested for binding to immobilized HEp2 cells (a) and by ELISA for binding against polyspecific reagents (PSR) including Chinese hamster ovary cells solubilized membrane protein (CHO-SMP), insulin and single-strand DNA (ssDNA) (b). For HEp2 assay, immunofluorescence showed binding of antibodies to immobilized HEp2 cells was detected by FITC-labelled secondary antibody. Fluorescent intensity from strong to weak were labeled as "++", "+" and "+/-" accordingly. "-" indicated little or no signal could be observed. Positive and negative controls for the HEp2 assay are provided by the manufacturer. In PSR ELISA, Bococizumab which is a humanized mAb targeting the LDL receptor-binding domain of PCSK9 and studied in phase I-III clinical studies (1), was used as a positive control. The color curves indicate antibodies that can react with PSR, while gray curves are the antibodies with little or no binding to PSR. DEN3 mAb was used as a negative control. 
a

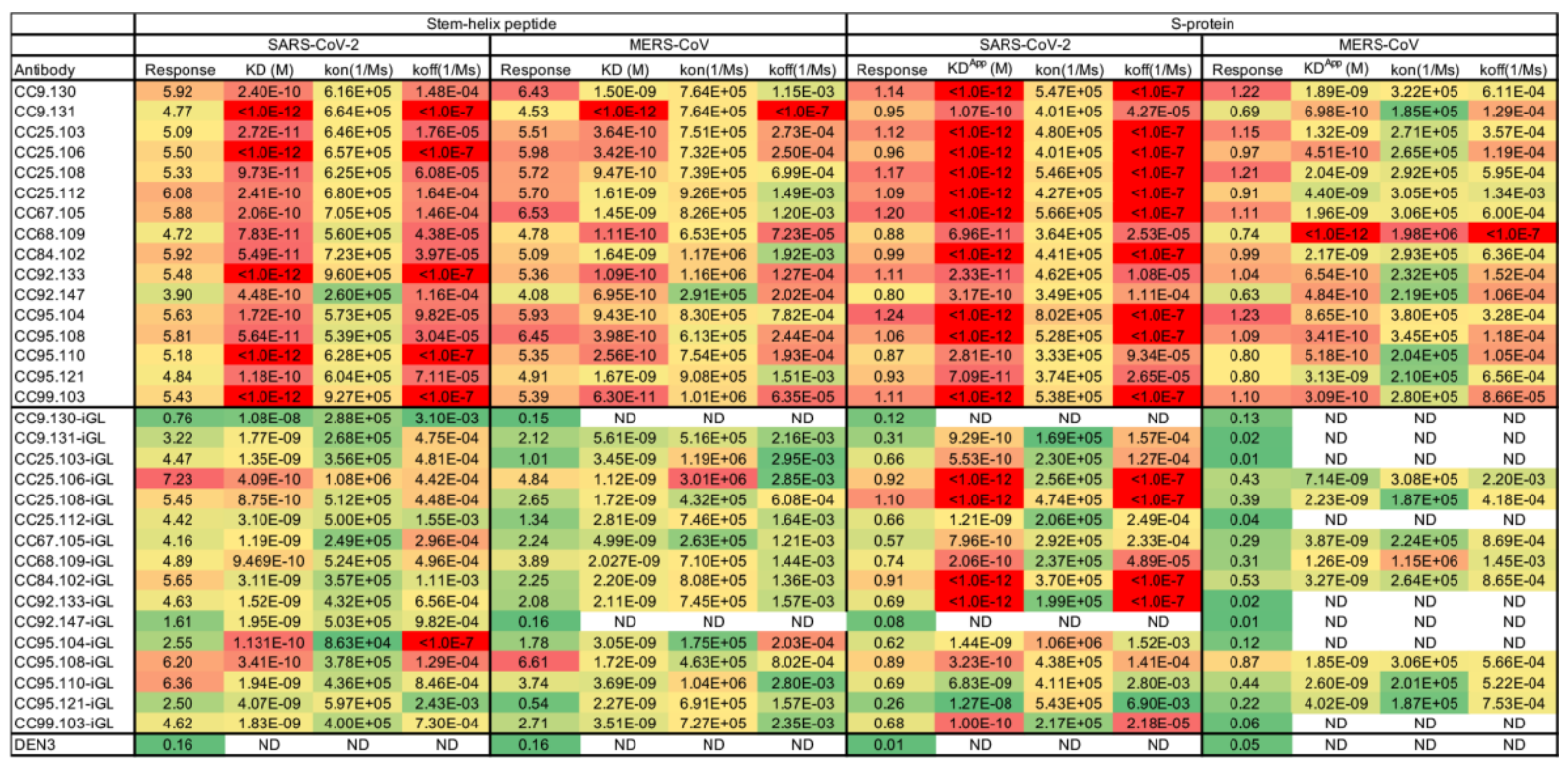

b
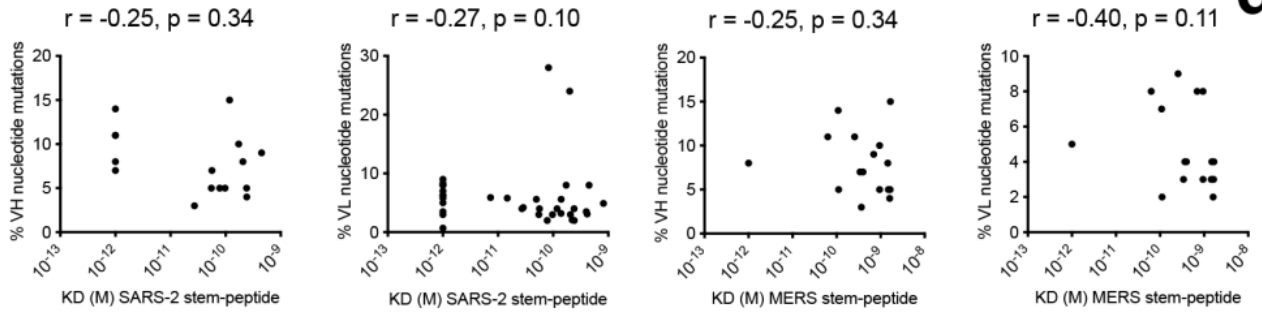

$r=0.49, p=0.05$
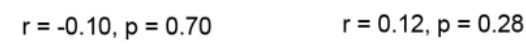

$r=-0.40, p=0.12$
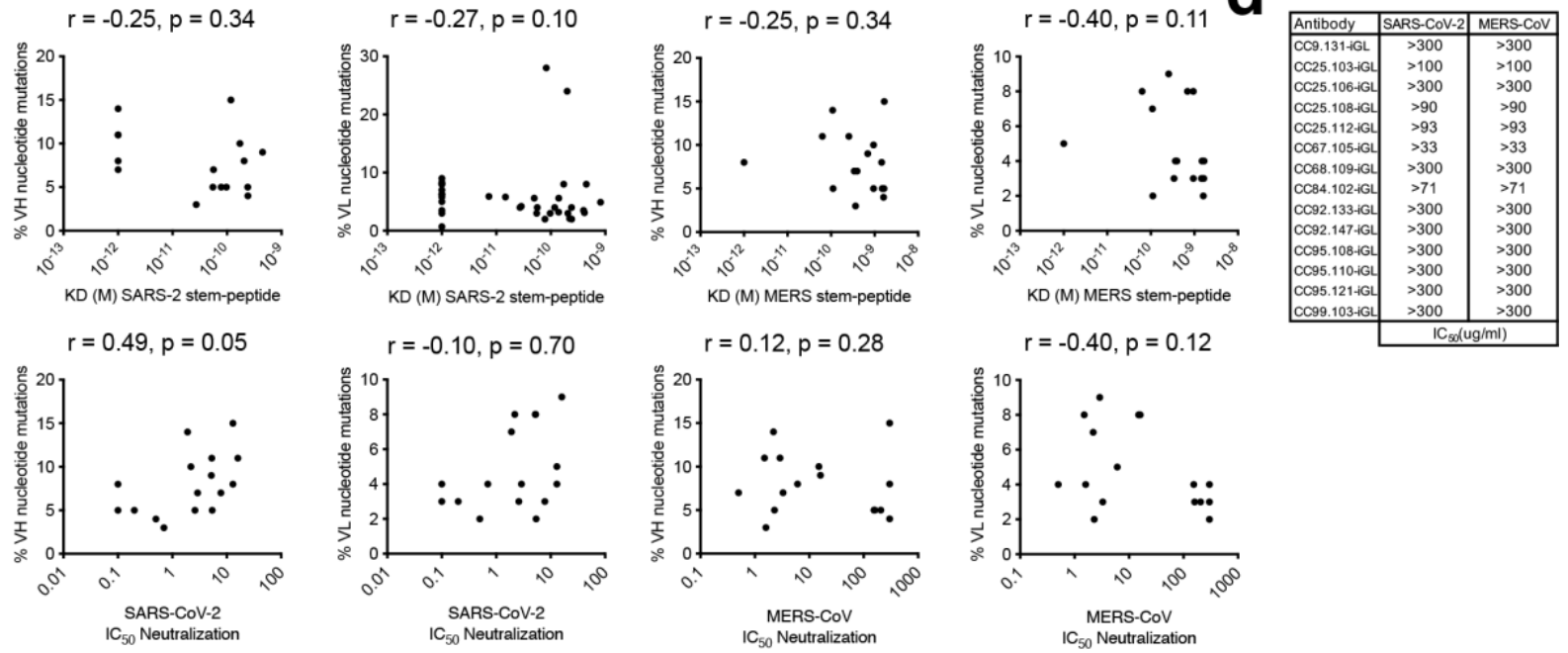

C

$r=0.64, p=0.009$

$$
r=-0.44, p=0.08
$$

$r=0.79, p<0.0004$
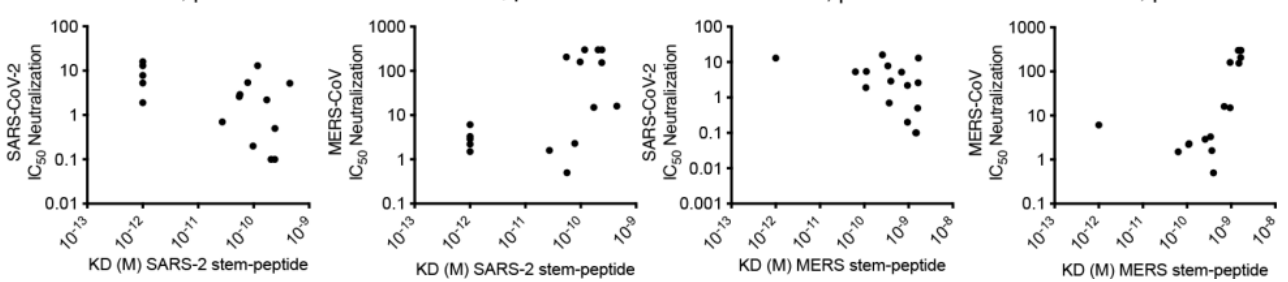
SHMs and Neutralization. a. BioLayer Interferometry (BLI) binding kinetics of 16 S2 stem-helix bnAbs and their inferred germline (iGL) Ab versions with SARS-CoV-2 and MERS-CoV stem-helix peptides and S-proteins. Binding kinetics were obtained using the 1:1 binding kinetics fitting model on ForteBio Data Analysis software and maximum binding responses, dissociations constants $\left(K_{D}\right)$ and on-rate $\left(K_{o n}\right)$ and off-rate constants $\left(k_{o f f}\right)$ for each antibody peptide interaction are shown. $K_{D}$, $k_{\text {on }}$ and $k_{\text {off }}$ values were calculated only for antibody-antigen interactions where a maximum binding response of 
$10390.2 \mathrm{~nm}$ was obtained. MAbs were also tested with SARS-CoV-2 and MERS-CoV S1040 proteins and the responses, apparent binding constants $\left(K_{D}{ }^{A p p}\right)$ and $k_{o n}$ and $k_{\text {off }}$ constants 1041 for each antibody-antigen interaction are indicated. The iGL Ab versions of stem-helix 1042 bnAbs showed reduced binding compared their mature versions. b. Correlations of stem1043 helix mAb binding ( $K_{\mathrm{D}}(\mathrm{M})$ values) to SARS-CoV-2 and MERS-CoV peptides and virus 1044 neutralization with heavy (VH) chain and light (VL) chain SHM levels. c. Correlations of 1045 stem-helix mAb binding ( $K_{\mathrm{D}}(\mathrm{M})$ values) to SARS-CoV-2 and MERS-CoV peptides with 1046 neutralization against their corresponding viruses. Correlations were determined by 1047 nonparametric Spearman correlation two-tailed test with 95\% confidence interval. The 1048 Spearman correlation coefficient ( $r$ ) and $p$-value are indicated. d. IC 50 neutralization of S2 1049 stem-helix bnAb iGLs with SARS-CoV-2 and MERS-CoV. 


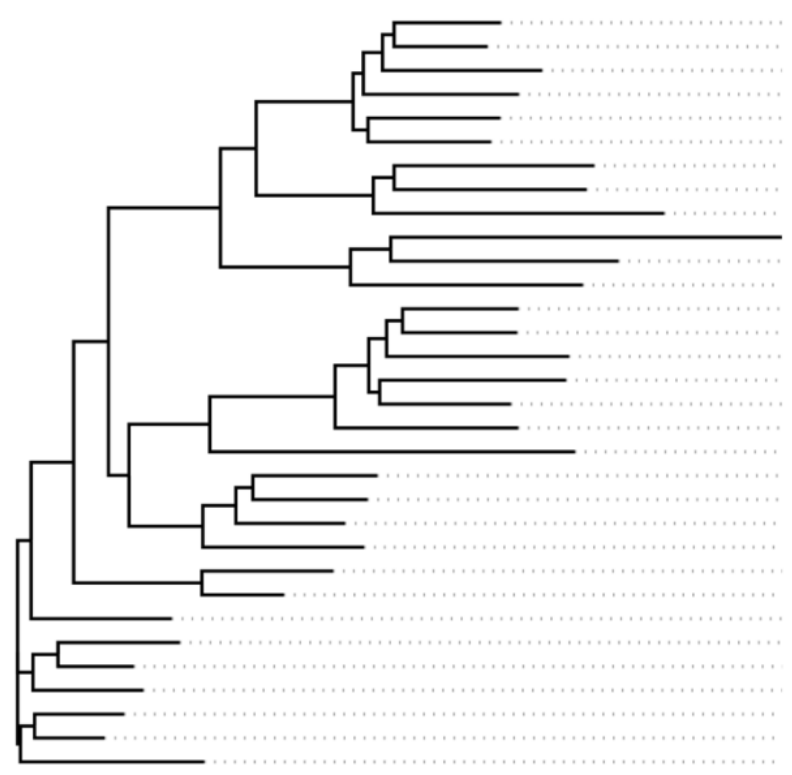

VL gene

CDRL3 has WD motif
VH gene

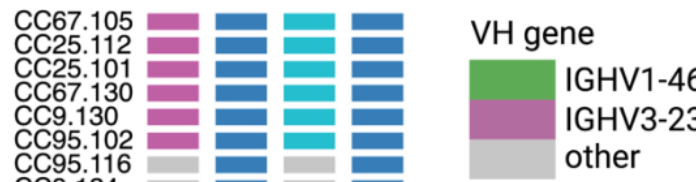

CDRH/L3 has motif

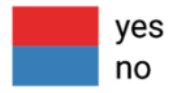

VL gene

IGKV3-20

IGLV1-51

other
Supplementary Figure 6. Immunogenetics analysis of heavy and light chain sequences of $\mathbf{3 2}$ unique $\mathbf{S 2}$ stem-helix mAbs. The phylogenetic tree represents concatenated heavy and light chain amino acid sequences of 32 S2 stem-helix mAbs. mAbs IDs are shown on the right. Four colored columns on the right show the following characteristics of mAbs (from left to right): (1) the germline $\mathrm{V}$ gene of each heavy chain (IGHV1-46: green, IGHV3-23: plum, others: gray), (2) the presence of RG motif in the amino acid sequence of each CDRH3 (motif is present: red, motif is missing: blue), (3) the germline $V$ gene of each light chain (IGKV3-20: sky, IGLV1-51: cantaloupe, others: gray), (4) the presence of WD motif in the amino acid sequence of each CDRL3 (motif is present: red, motif is missing: blue). 
bioRxiv preprint doi: https://doi.org/10.1101/2022.03.04.479488; this version posted March 7, 2022. The copyright holder for this preprint (which was not certified by peer review) is the author/funder, who has granted bioRxiv a license to display the preprint in perpetuity. It is made available under aCC-BY-NC-ND 4.0 International license.

1062

1063

1064

1065

1066

1067

1068

1069

1070

1071

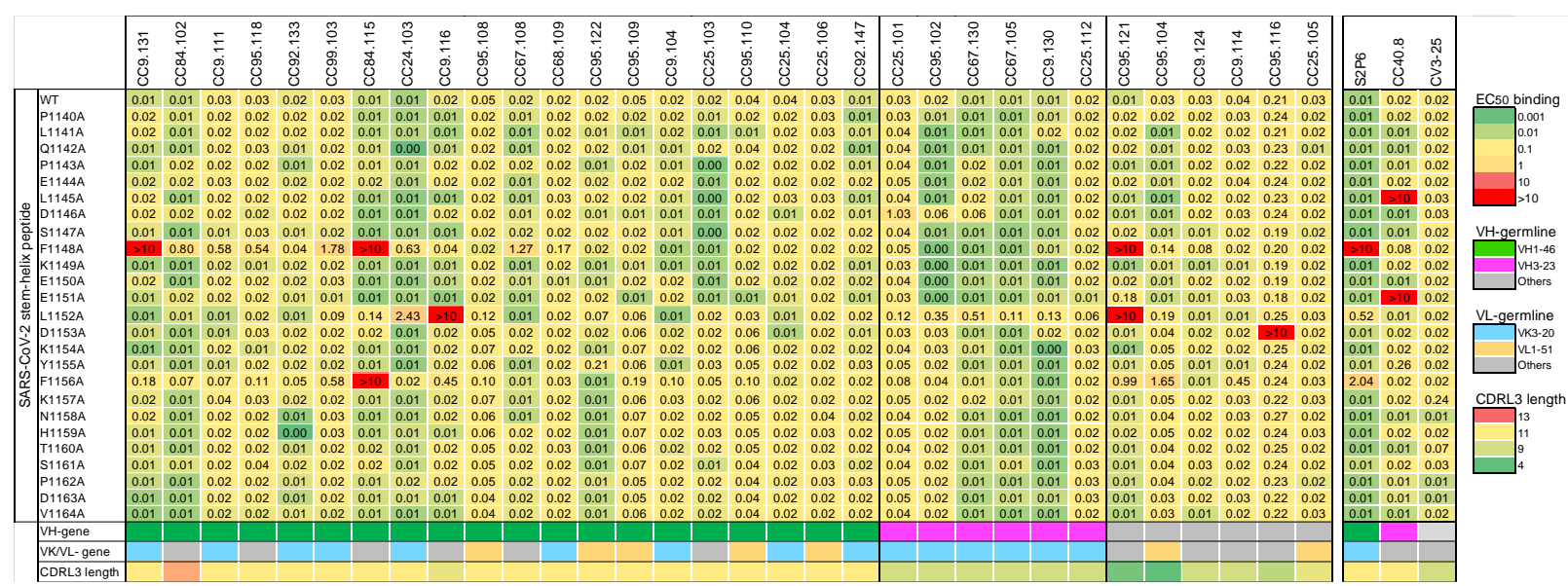

Supplementary Figure 7. Epitope mapping of S2 stem-helix bnAbs with SARS-CoV2 stem-helix peptide alanine scan mutants. Heatmap showing EC50 ELISA binding titers of S2-stem helix bnAbs to 25mer SARS-CoV-2 stem-helix peptide and its alanine scan mutants. Three hydrophobic residues, $\mathrm{F}^{1148}$, $\mathrm{L}^{1152}$ and $\mathrm{F}^{1156}$ were commonly targeted by stem-helix bnAbs. S2 stem-helix bnAbs are grouped based on their heavy chain gene usage (IGHV1-46, IGHV3-23 and others). The light chain germline genes (IGKV3-20, IGLV1-51 and other) and CDRL3 lengths are shown. S2P6, CC40.8 and CV3-25 S2 stemhelix mAbs were used as controls. 
a

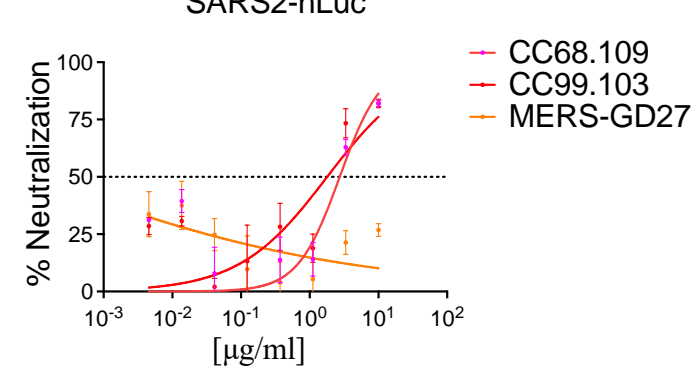

b

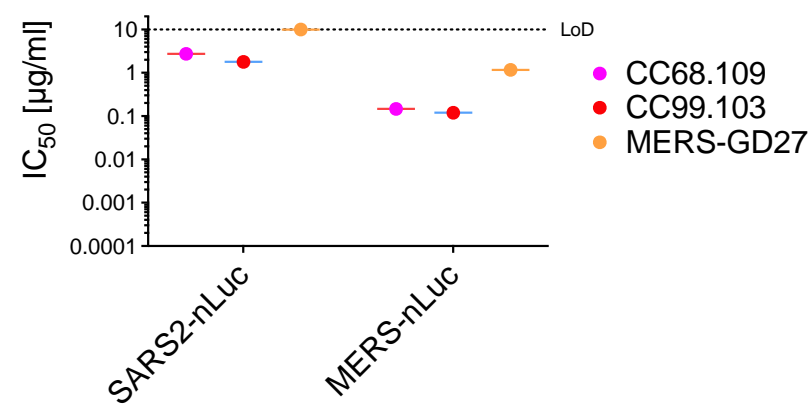

1072

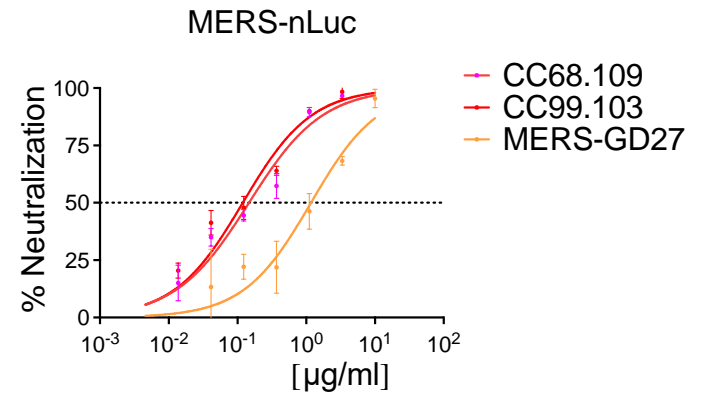

C Pseudoviruses

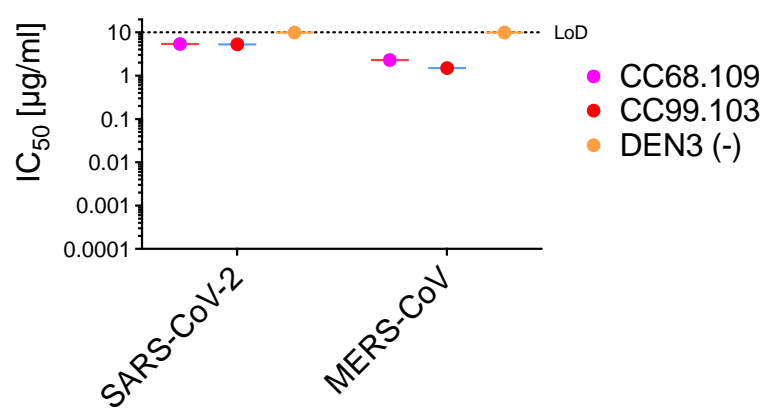

Supplementary Figure 8 . Neutralization of replication competent betacoronaviruses by select S2-stem helix bnAbs. a. Neutralization of replication competent viruses encoding SARS-CoV-2 (SARS2-nLuc), and MERS-CoV (MERS-nLuc) by 2 select S2 stem-helix bnAbs, CC68.109, and CC99.103. MERS-GD27 antibody (2) was a positive control for the MERS-CoV neutralization assay. b-c. Comparison of IC 50 neutralization titers of S2 stem-helix bnAbs with replication-competent (b) and pseudoviruses (c) of SARS-CoV-2 and MERS-CoV. 
bioRxiv preprint doi: https://doi org/10.1101/2022 03.04.479488; this version posted March 7, 2022. The copyright holder for this preprint (which was not certified by peer review) is the author/funder, who has granted bioRxiv a license to display the preprint in perpetuity. It is made available under aCC-BY-NC-ND 4.0 International license.

\section{References}

1082

1083

1. E. Q. Wang et al., Assessing the Potential Risk of Cross-Reactivity Between Anti-

1084

1085

1086

1087

1088 Bococizumab Antibodies and Other Anti-PCSK9 Monoclonal Antibodies. BioDrugs 33, 571-579 (2019).

2. P. Niu et al., Ultrapotent Human Neutralizing Antibody Repertoires Against Middle East Respiratory Syndrome Coronavirus From a Recovered Patient. J Infect Dis 218, 12491260 (2018). 